MIT-CTP 4063

TUM-EFT 3/09

arXiv:0911.4980

May 30, 2018

\title{
Quark Fragmentation within an Identified Jet
}

\author{
Massimiliano Procura ${ }^{1,2}$ and Iain W. Stewart ${ }^{1}$ \\ ${ }^{1}$ Center for Theoretical Physics, Laboratory for Nuclear Science, \\ Massachusetts Institute of Technology, Cambridge, MA 02139 \\ ${ }^{2}$ Physik-Department, Technische Universität München, D-85748 Garching, Germany
}

\begin{abstract}
We derive a factorization theorem that describes an energetic hadron $h$ fragmenting from a jet produced by a parton $i$, where the jet invariant mass is measured. The analysis yields a "fragmenting jet function" $\mathcal{G}_{i}^{h}(s, z)$ that depends on the jet invariant mass $s$, and on the energy fraction $z$ of the fragmentation hadron. We show that $\mathcal{G}_{i}^{h}$ can be computed in terms of perturbatively calculable coefficients, $\mathcal{J}_{i j}(s, z / x)$, integrated against standard non-perturbative fragmentation functions, $D_{j}^{h}(x)$. We also show that $\sum_{h} \int d z z \mathcal{G}_{i}^{h}(s, z)$ is given by the standard inclusive jet function $J_{i}(s)$ which is perturbatively calculable in QCD. We use Soft-Collinear Effective Theory and for simplicity carry out our derivation for a process with a single jet, $\bar{B} \rightarrow X h \ell \bar{\nu}$, with invariant mass $m_{X h}^{2} \gg \Lambda_{\mathrm{QCD}}^{2}$. Our analysis yields a simple replacement rule that allows any factorization theorem depending on an inclusive jet function $J_{i}$ to be converted to a semi-inclusive process with a fragmenting hadron $h$. We apply this rule to derive factorization theorems for $\bar{B} \rightarrow X K \gamma$ which is the fragmentation to a Kaon in $b \rightarrow s \gamma$, and for $e^{+} e^{-} \rightarrow$ (dijets) $+h$ with measured hemisphere dijet invariant masses.
\end{abstract}




\section{INTRODUCTION}

Factorization theorems are crucial for applying QCD to hard scattering processes involving energetic hadrons or identified jets. In single inclusive hadron production, an initial energetic parton $i=\{u, d, g, \bar{u}, \ldots\}$ produces an energetic hadron $h$ and accompanying hadrons $X$. Factorization theorems for these fragmentation processes have been derived at leading power for high-energy $e^{+} e^{-} \rightarrow X h$,

$$
d \sigma=\sum_{i} d \hat{\sigma}_{i} \otimes D_{i}^{h}
$$

as well as lepton-nucleon deeply inelastic scattering, $e^{-} p \rightarrow e^{-} X h$,

$$
d \sigma=\sum_{i j} d \hat{\sigma}_{i j} \otimes D_{i}^{h} \otimes f_{j / p}
$$

For a factorization review see Ref. [1]. In Eqs. (11) and (2) the cross sections are convolutions of perturbatively calculable hard scattering cross sections, $d \hat{\sigma}$, with non-perturbative but universal fragmentation functions $D_{i}^{h}(z)$, and parton distributions $f_{j / p}(\xi)$. The fragmentation functions $D_{i}^{h}(z)$ encode information on how a parton $i$ turns into the observed hadron $h$ with a fraction $z$ of the initial parton large momentum. Fragmentation functions are also often used for processes where a complete proof of factorization is still missing, such as high-energy hadron-hadron collision, $H_{1} H_{2} \rightarrow h X$.

Another interesting class of hard scattering processes are those with identified jets. Examples include dijet production $e^{+} e^{-} \rightarrow X_{J_{1}} X_{J_{2}} X_{s}$ where $X_{J_{1,2}}$ are two jets of hadrons, and $X_{s}$ denotes soft radiation between the jets. If we measure an inclusive event shape variable such as thrust, or hemisphere invariant masses, then the cross section for this dijet process has the leading order factorization theorem [2 [5]

$$
d \sigma=H_{2 \text { jet }} J \otimes J \otimes S_{2 \text { jet }}
$$

Here the $J=J(s)$ are inclusive jet functions depending on a jet invariant mass variable $s$, $S_{2 j e t}$ is a soft function which gets convoluted with the $J_{\mathrm{S}}$ as denoted by $\otimes$, and $H_{2 \text { jet }}$ is a multiplicative hard coefficient. Another example of this type is the $\bar{B} \rightarrow X_{u} \ell \bar{\nu}_{\ell}$ decay in a region of phase space where $X_{u}$ is jet-like $\left(\Lambda_{\mathrm{QCD}} \ll m_{X_{u}}^{2} \ll m_{B}^{2}\right)$. Here the leading order factorization theorem for the decay rate is [6, 7]

$$
d \Gamma=H J_{1} \otimes S,
$$

with a hard function $H$ for the underlying $b \rightarrow u \ell \bar{\nu}_{\ell}$ process, the same inclusive jet function $J$ as in the previous example, and a "shape function" $S$ which is the parton distribution for a $b$-quark in the $B$-meson in the heavy quark limit.

In this paper we will analyze processes which combine the above two cases, namely both the fragmentation of a hard parton $i$ into $h$ and the measurement of a jet invariant mass. 
Since this probes fragmentation at a more differential level, we expect it can teach us interesting things about the jet dynamics involved in producing $h$, and shed light on the relative roles of perturbative partonic short-distance effects and non-perturbative hadronization. We derive factorization theorems that depend on a new "fragmenting jet function " $\mathcal{G}_{i}^{h}(s, z)$. This function depends on $s$, the jet invariant mass variable, and on $z$, the ratio of the large light-like momenta of the fragmentation hadron and parton. Two interesting formulae involving $\mathcal{G}_{i}^{h}$ will be derived. The first formula states that

$$
J_{i}(s, \mu)=\frac{1}{2} \sum_{h} \int \frac{d z}{(2 \pi)^{3}} z \mathcal{G}_{i}^{h}(s, z, \mu),
$$

so that the inclusive jet-function can be decomposed into a sum of terms, $\mathcal{G}_{i}^{h}$, for fragmentation to a hadron $h$ with $m_{h}^{2} \ll m_{X}^{2}$. This formula also leads to a replacement rule for factorization theorems, where we can take any process involving an inclusive jet function, and replace $J_{i} \rightarrow \mathcal{G}_{i}^{h}$ to obtain the corresponding process with a fragmenting jet.

The second formula states that to leading order in $\Lambda_{\mathrm{QCD}}^{2} / s \ll 1$ we have

$$
\mathcal{G}_{i}^{h}(s, z, \mu)=\sum_{j} \int \frac{d x}{x} \mathcal{J}_{i j}\left(s, \frac{z}{x}, \mu\right) D_{j}^{h}(x, \mu),
$$

so that the fragmenting jet function can be expressed in terms of perturbatively calculable coefficients $\mathcal{J}_{i j}$, together with the standard unpolarized fragmentation functions $D_{j}^{h}(x, \mu)$ renormalized in the $\overline{\mathrm{MS}}$ scheme.

To introduce the concept of $\mathcal{G}_{i}^{h}$ and study its properties, we will specialize to a process with a single jet recoiling against leptons, namely $\bar{B} \rightarrow X h \ell \bar{\nu}_{\ell}$. Using Soft-Collinear Effective Theory (SCET) [7-10] we derive leading-order factorization formulae for $\bar{B} \rightarrow X h \ell \bar{\nu}_{\ell}$ decay rates, in the region of phase space characterized by $\Lambda_{\mathrm{QCD}}^{2} \ll m_{X h}^{2} \ll m_{B}^{2}$ where the hadronic final state is jet-like, and where the energetic hadron $h$ fragments from the jet. This $b \rightarrow u \ell \bar{\nu}_{\ell}$ process has the virtue of having a single jet whose invariant mass can be measured in a straightforward manner with available $B$-factory data. Despite our focus on $\bar{B} \rightarrow X h \ell \bar{\nu}_{\ell}$ the results obtained can be immediately generalized to fragmentation in other processes where a jet invariant mass measurement is made. Two examples will be described.

The paper is organized as follows. In Sec. II we review the standard definition of the quark fragmentation function $D_{q}^{h}(z)$ and highlight features that are relevant for later parts of our analysis. Sec. IIII is devoted to the process $\bar{B} \rightarrow X h \ell \bar{\nu}_{\ell}$, including a discussion of kinematics in Sec. IIIA. Results for relevant differential decay rates in terms of components of an appropriate hadronic tensor are given in Sec. IIIB, Sec. IV contains the derivation of the SCET factorization formulae for $\bar{B} \rightarrow X h \ell \bar{\nu}_{\ell}$, and the definition of the "fragmenting jet function " $\mathcal{G}_{i}^{h}$. In Sec. $\nabla$ we discuss the relations shown above in Eqs. (5) and (6). Conclusions, outlook, and the generalization to other processes are given in Sec. VII. 


\section{THE FRAGMENTATION FUNCTION $D(z)$}

Defining $n^{\mu}=(1,0,0,1)$ and $\bar{n}^{\mu}=(1,0,0,-1)$, the light-cone components of a generic four-vector $a^{\mu}$ are denoted by $a^{+}=n \cdot a$ and $a^{-}=\bar{n} \cdot a$ where $n^{2}=\bar{n}^{2}=0$ and $n \cdot \bar{n}=2$. With $a_{\perp}^{\mu}$ we indicate the components of $a^{\mu}$ orthogonal to the plane spanned by $n^{\mu}$ and $\bar{n}^{\mu}$. For energetic collinear particles we will follow the convention where the large momentum is $p^{-}$and the small momentum is $p^{+}$.

Let us consider a quark $q$ with momentum $k^{\mu}$ fragmenting to an observed hadron $h$ with momentum $p^{\mu}$. In a frame where $\vec{k}_{\perp}=0$, the hadron has $p_{h}^{-} \equiv z k^{-}$and $p_{h}^{+}=\left(\vec{p}_{h}^{\perp}+m_{h}^{2}\right) / p_{h}^{-}$. The standard unpolarized fragmentation function $D_{i}^{h}(z)$ is defined as the integral over $p_{h}^{\perp}$ of the "probability distribution" that the parton $i$ decays into the hadron $h$ with momentum $p_{h}^{\mu}$ [11, 12], see also [13-16]. With the gauge choice $\bar{n} \cdot A=0$, the unrenormalized quark fragmentation function has the following operator definition [11]:

$$
D_{q}^{h}(z)=\left.\frac{1}{z} \int \mathrm{d}^{2} p_{h}^{\perp} \int \frac{\mathrm{d} x^{+} \mathrm{d}^{2} x_{\perp}}{2(2 \pi)^{3}} e^{i k^{-} x^{+} / 2} \frac{1}{4 N_{c}} \operatorname{Tr} \sum_{X}\left\langle 0\left|\not h \psi\left(x^{+}, 0, x_{\perp}\right)\right| X h\right\rangle\langle X h|\bar{\psi}(0)| 0\rangle\right|_{p_{X}^{\perp}=0},
$$

where $\psi$ is the quark field quantized on $x^{-}=0, N_{c}=3$ is the number of colors, and the trace is taken over color and Dirac indices. In Eq. (17) the state $|X h\rangle=\left|X h\left(p_{h}\right)\right\rangle$ has a hadron $h$ with momentum $p_{h}=\left(p_{h}^{-}, \vec{p}_{h}^{\perp}\right)$, and an average over polarizations of $h$ is assumed. Boost invariance along the non- $\perp$ direction implies that $D_{q}^{h}$ can only be a function of $z=p_{h}^{-} / k^{-}$ and not $k^{-}$or $p_{h}^{-}$individually.

Performing a rotation and a boost to a frame where $\vec{p}_{h}^{\perp}=0$ with $p_{h}^{-}$left unchanged, $\vec{k}_{\perp}$ becomes $-\vec{p}_{h}^{\perp} / z$, and Eq. (77) can be written in a gauge-invariant form as [11]

$$
D_{q}^{h}(z)=\left.z \int \frac{\mathrm{d} x^{+}}{4 \pi} e^{i k^{-} x^{+} / 2} \frac{1}{4 N_{c}} \operatorname{Tr} \sum_{X}\left\langle 0\left|\not h \Psi\left(x^{+}, 0,0_{\perp}\right)\right| X h\right\rangle\langle X h|\bar{\Psi}(0)| 0\rangle\right|_{p_{h}^{\perp}=0},
$$

where the field $\Psi\left(x^{+}\right)=\Psi\left(x^{+}, 0,0_{\perp}\right)$ contains an anti path-ordered Wilson line of gluon fields, in a $\overline{3}$ representation

$$
\Psi\left(x^{+}\right) \equiv \psi\left(x^{+}\right)\left[\overline{\mathrm{P}} \exp \left(i g \int_{x^{+}}^{+\infty} \mathrm{d} s \bar{n} \cdot A^{T}(s \bar{n})\right)\right] .
$$

We note that the form of Eq. (8) is not altered if we perform a Lorentz transformation to a frame where $\vec{p}_{h}^{\perp}$ equals an arbitrary fixed reference value $\vec{p}_{\perp}^{\text {ref }}$. In this case $\vec{k}_{\perp}=\left(\vec{p}_{\perp}^{\text {ref }}-\vec{p}_{h}^{\perp}\right) / z$ and $\vec{p}_{\perp}^{\text {ref }}$ does not play any role due to the integrals over $p_{h}^{\perp}$ and $x_{\perp}$ in Eq. (7).

Our knowledge of the fragmentation functions is anchored to the use of factorization theorems to describe measurements of single-inclusive high-energy processes. Constraints are obtained by using perturbative results for the partonic hard collision as input. For example, writing out the complete form of Eq. (11) for single-inclusive $e^{+} e^{-}$annihilation into 
a specific hadron $h$ at center-of-mass energy $E_{\mathrm{cm}}$, we have

$$
\frac{1}{\sigma_{0}} \frac{d \sigma^{h}}{d z}\left(e^{+} e^{-} \rightarrow h X\right)=\sum_{i} \int_{z}^{1} \frac{d x}{x} C_{i}\left(E_{\mathrm{cm}}, x, \mu\right) D_{i}^{h}(z / x, \mu),
$$

where $\sigma_{0}$ is the tree level cross section for $e^{+} e^{-} \rightarrow$ hadrons, $\mu$ is the renormalization scale in the $\overline{\mathrm{MS}}$ scheme, and $C_{i}$ is the coefficient for the short-distance partonic process producing the parton $i$. In Eq. (10) the sum includes the contributions from the different parton types, $i=u, \bar{u}, d, g \ldots$ and the $C_{i}$ 's are calculable in perturbation theory, so measurements of $d \sigma^{h} / d z$ constrain $D_{i}^{h}$.

Model parameters for fragmentation functions have been extracted by fitting to cross section data for single charged hadron inclusive $e^{+} e^{-}$annihilation, including high statistics measurements at CERN-LEP and SLAC [17 20]. More recently, these data have been combined with semi-inclusive lepton-nucleon deeply inelastic and $p p$ cross sections from HERMES and RHIC experiments, respectively, to perform a global analysis of pion, kaon and (anti-)proton fragmentation based on the factorized expressions for the relevant cross sections, with partonic input at next-to-leading order in QCD perturbation theory [21, 22], see also [23]. These analyses confirm the universal nature of the fragmentation function, and, for the $\pi^{+}$, constrain the fragmentation model for the dominant $D_{u}^{\pi}(z)$ with uncertainties at the $10 \%$ level for $z \gtrsim 0.5$ [20]. There is less sensitivity to gluon fragmentation functions, and, correspondingly, these have larger uncertainties.

Factorization theorems like the one in Eq. (10) have been proven to all orders in $\alpha_{s}$ at leading order in $\Lambda_{\mathrm{QCD}} / E_{\mathrm{cm}}$ for processes in which all Lorentz invariants like $E_{\mathrm{cm}}^{2}=$ $\left(p_{e^{+}}+p_{e^{-}}\right)^{2}$ are large and comparable, except for particle masses [1]. The original proofs are based on the study of the analytic structure of Feynman diagrams and on a powercounting method to find the strength of infrared singularities in massless perturbation theory. Factorization is possible because only a limited set of regions in the space of loop and final state momenta contribute to leading power, namely the so-called leading regions which are hard, collinear, and soft. For processes involving fragmentation, the leading regions contain a jet subdiagram that describes the jet in which the hadron $h$ is observed [1], see also [11, 24 26]. Accordingly, the fragmentation function that can be constrained by applying factorization at leading power, corresponds to Eq. (17) only because the sum over $X$ is dominated by jet-like configurations for the $|X h\rangle$ states. Therefore, it is interesting to explore whether more can be learned about the fragmentation process when additional measurements are made on the accompanying jet.

Here we consider what amounts to the simplest additional measurement, namely that of the jet invariant mass $m_{X h}^{2}=\left(p_{X}+p_{h}\right)^{2}$. Rather than using classic techniques we exploit the powerful computational framework of SCET. 


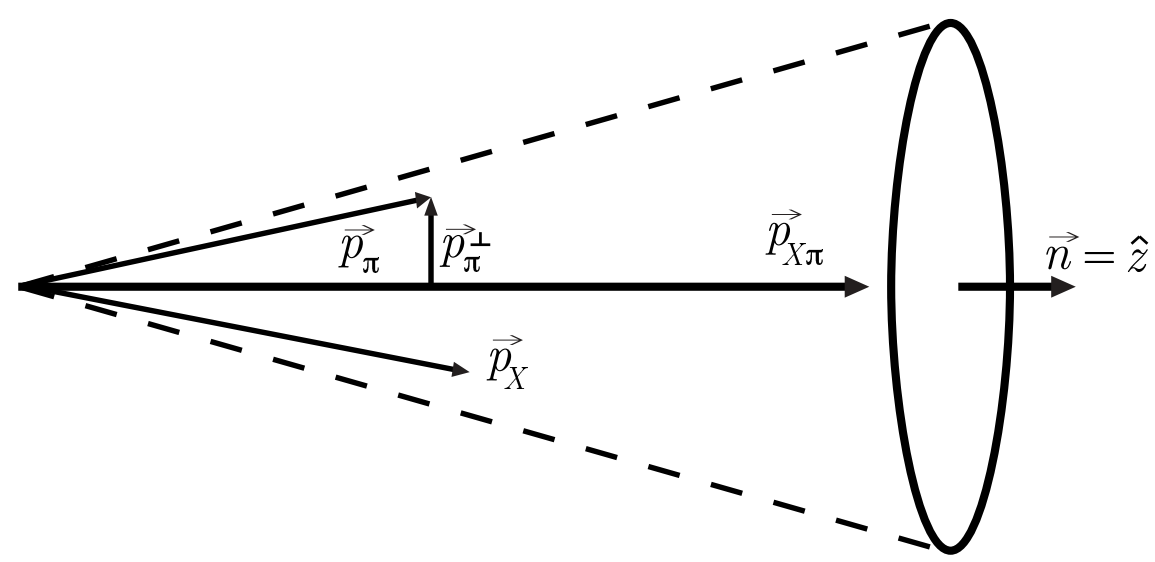

FIG. 1: Kinematic configuration for a pion fragmenting from a jet $X_{u} \rightarrow X \pi$.

\section{FRAGMENTATION FROM AN IDENTIFIED JET IN $\bar{B} \rightarrow X h \ell \bar{\nu}$}

Consider the weak transition $b \rightarrow u \ell \bar{\nu}_{\ell}$ measured with inclusive decays $\bar{B} \rightarrow X_{u} \ell \bar{\nu}_{\ell}$. The phase space region where $X_{u}$ is jet-like plays an important role, because the experimental cuts which remove $b \rightarrow c$ background most often restrict the final state to this region. Experimentally there is exquisite control over this process, e.g. in a large sample of events the neutrino momentum has been reconstructed by determining the recoil momentum of the $\bar{B}$, and the spectrum is available for the jet invariant mass $m_{X_{u}}^{2}[27-29]$. There has also been an extensive amount of theoretical work on this process based on the factorization theorem shown in Eq. (41) [2, 7, 30 38]. From our perspective the nice thing about $\bar{B} \rightarrow X_{u} \ell \bar{\nu}_{\ell}$ is that it involves only a single jet, and hence provides the simplest possible framework to extend the factorization analysis involving jet functions to the fragmentation process we are interested in, where $X_{u} \rightarrow(X h)_{u}$. Here $h$ is a light-hadron fragmenting from a $u$-quark, with $m_{h} \ll m_{B}$. Without any loss of generality, we shall refer to $h$ as a pion $\pi$ for the following few sections, though we will return to the general notation $h$ at the end.

\section{A. Kinematics}

In the $\bar{B}$ rest frame, the inclusive process $\bar{B} \rightarrow X_{u} \ell \bar{\nu}_{\ell}$ can be completely described by three variables, often taken as the hadronic invariant mass $m_{X_{u}}^{2}$, the square of the total leptonic momentum $q^{2}$ (with $q^{\mu}=p_{\ell}^{\mu}+p_{\bar{\nu}}^{\mu}$ ), and the charged lepton energy $E_{\ell}$. In the jetlike region a more convenient set of variables is $p_{X_{u}}^{+}, p_{X_{u}}^{-}$, and $E_{\ell}$ where $q^{\mu}$ is aligned with the $-\hat{z}$-axis, and hence the jet-axis is along $+\hat{z}$ with $p_{X_{u}}^{\mp}=E_{X_{u}} \pm\left|\vec{p}_{X_{u}}\right|$.

With an identified hadron in the final state, $\bar{B} \rightarrow X \pi \ell \bar{\nu}_{\ell}$, there are three additional kinematic variables corresponding e.g. to the three independent components of $\vec{p}_{\pi}$. The orientation of the spatial axes will still be chosen such that $p_{X \pi}^{\perp \mu}=0$, as shown in Fig. 11. In this frame the perpendicular component of the total lepton momentum vanishes, $q_{\perp}^{\mu}=0$, and $p_{B}^{\mu}=m_{B} v^{\mu}$ with $v^{\mu}=\left(n^{\mu}+\bar{n}^{\mu}\right) / 2$. The six independent kinematic variables that we 
will use to characterize this semi-inclusive process are: $p_{X \pi}^{+}, p_{X \pi}^{-}, E_{\ell}, p_{\pi}^{-}, p_{\pi}^{+}, \phi_{\ell}$, where $\phi_{\ell}$ denotes the azimuthal angle of the lepton with respect to the $z$-axis and $p_{\pi}^{\mp}=E_{\pi} \pm p_{\pi}^{z}$. By definition we have $p_{X \pi}^{+} \leq p_{X \pi}^{-}, E_{\pi}=\left(p_{\pi}^{+}+p_{\pi}^{-}\right) / 2$ and $p_{\pi}^{+}=\left(\vec{p}_{\pi}^{\perp 2}+m_{\pi}^{2}\right) / p_{\pi}^{-}$. In this section we carry out a complete analysis of the kinematics, determining the phase space limits for the six kinematic variables without imposing any added restrictions or assumptions. (Later in Section [V we will specialize to the case of a fragmentation pion collinear in the $\vec{n}$ direction with $p_{\pi}^{+} \leq p_{\pi}^{-}$.) Note that $m_{X \pi}^{2}=p_{X \pi}^{-} p_{X \pi}^{+}$so for our process the measurement of the jetinvariant mass is a measurement of the invariant mass of all final state hadronic particles. Lepton masses will be neglected throughout, but the hadron mass $m_{\pi}^{2}$ will be kept for all calculations involving kinematics.

For three of our six variables we can treat $|X \pi\rangle$ as a combined state $\left|X_{u}\right\rangle$, and hence $\left\{p_{X \pi}^{+}, p_{X \pi}^{-}, E_{\ell}\right\}$ have the same limits as in the inclusive case, and are given by ${ }^{1}$

$$
m_{\pi} \leq p_{X \pi}^{-} \leq m_{B}, \quad \frac{m_{\pi}^{2}}{p_{X \pi}^{-}} \leq p_{X \pi}^{+} \leq p_{X \pi}^{-}, \quad \frac{m_{B}-p_{X \pi}^{-}}{2} \leq E_{\ell} \leq \frac{m_{B}-p_{X \pi}^{+}}{2} .
$$

In order to determine the limits for the remaining variables, let us first consider a frame where $\vec{p}_{X \pi}=0$, so that it is as if we have $X_{u} \rightarrow X \pi$ decay in the $X_{u}$ rest frame. We denote the quantities evaluated in this frame by a '*'. Since

$$
E_{\pi}^{*}=\frac{m_{X \pi}^{2}+m_{\pi}^{2}-p_{X}^{2}}{2 m_{X \pi}}
$$

the constraint $p_{X}^{2} \geq 0$ implies

$$
m_{\pi} \leq E_{\pi}^{*} \leq \frac{m_{X \pi}^{2}+m_{\pi}^{2}}{2 m_{X \pi}}
$$

Furthermore, in this $X_{u}$ rest frame there are no restrictions on the azimuthal angle $\phi_{\pi}^{*}$ of the pion with respect to the $z$-axis, nor of that of the charged lepton $\phi_{\ell}^{*}$, i.e. $0 \leq \phi_{\pi}^{*}$, $\phi_{\ell}^{*} \leq 2 \pi$. The polar angle of the pion is also unconstrained:

$$
0 \leq \theta_{\pi}^{*} \leq \pi
$$

Since $p_{\pi}^{+^{*}}+p_{\pi}^{-*}=2 E_{\pi}^{*}$, from Eq.(13)

$$
p_{\pi}^{+*} \leq m_{X \pi}+\frac{m_{\pi}^{2}}{m_{X \pi}}-p_{\pi}^{-*} .
$$

Furthermore, since $\left|\vec{p}_{\pi}^{\perp *}\right|^{2} \geq 0$,

$$
p_{\pi}^{+*} \geq \frac{m_{\pi}^{2}}{p_{\pi}^{-*}}
$$

\footnotetext{
$\overline{{ }^{1} \text { Table } 2}$ in Ref. [34] lists these limits for $\bar{B} \rightarrow X_{u} \ell \bar{\nu}$ for the six possible orders of integration.
} 
For the maximum $p_{\pi}^{*-}=p_{X \pi}^{*-}=m_{X \pi}$, these limits force $p_{\pi}^{*+}=m_{\pi}^{2} / m_{X \pi}$ and the pion travels along the $\hat{z}$ axis. (Interchanging $p_{\pi}^{*+} \leftrightarrow p_{\pi}^{*-}$ gives the case where the pion travels along $-\hat{z}$.) For $p_{\pi}^{*-}=p_{\pi}^{*+}=\left(m_{X \pi}+m_{\pi}^{2} / m_{X \pi}\right) / 2$, we have a pion traveling purely in the $\perp$-plane with maximal energy. Note that Eqs. (15) and (16) imply other limits such as $p_{\pi}^{+*} \leq\left|\vec{p}_{\pi}^{\perp *}\right|_{\max }^{2} / p_{\pi}^{-*}$, as well as $p_{\pi}^{+*} \leq p_{X \pi}^{+}{ }^{*}=m_{X \pi}$, and

$$
p_{\pi}^{+*}=E_{\pi}^{*}-\sqrt{E_{\pi}^{*^{2}}-m_{\pi}^{2}} \cos \theta_{\pi}^{*}
$$

for all $\cos \theta_{\pi}^{*}$. This holds true for both cases $p_{\pi}^{+*} \leq p_{\pi}^{-*}$ and $p_{\pi}^{+*} \geq p_{\pi}^{-*}$, which correspond, respectively, to $0 \leq \cos \theta_{\pi}^{*} \leq 1$ and $-1 \leq \cos \theta_{\pi}^{*} \leq 0$.

Let us now perform a boost along the $z$-axis with velocity $\vec{v}_{X \pi}=v_{X \pi} \hat{e}_{z}$ to the frame where the $B$-meson decays at rest, which requires

$$
v_{X \pi}=\frac{\sqrt{E_{X \pi}^{2}-m_{X \pi}^{2}}}{E_{X \pi}}=\frac{p_{X \pi}^{-}-p_{X \pi}^{+}}{p_{X \pi}^{-}+p_{X \pi}^{+}}
$$

where $0 \leq v_{X \pi}<1$. Boosting Eqs. (15) and (16) yields the final result for the $p_{\pi}^{ \pm}$phase space boundaries:

$$
\frac{m_{\pi}^{2}}{p_{X \pi}^{+}} \leq p_{\pi}^{-} \leq p_{X \pi}^{-}, \quad \frac{m_{\pi}^{2}}{p_{\pi}^{-}} \leq p_{\pi}^{+} \leq p_{X \pi}^{+}\left(1-\frac{p_{\pi}^{-}}{p_{X \pi}^{-}}\right)+\frac{m_{\pi}^{2}}{p_{X \pi}^{-}} .
$$

Equivalently, for the opposite order of integration,

$$
\frac{m_{\pi}^{2}}{p_{X \pi}^{-}} \leq p_{\pi}^{+} \leq p_{X \pi}^{+}, \quad \frac{m_{\pi}^{2}}{p_{\pi}^{+}} \leq p_{\pi}^{-} \leq p_{X \pi}^{-}\left(1-\frac{p_{\pi}^{+}}{p_{X \pi}^{+}}\right)+\frac{m_{\pi}^{2}}{p_{X \pi}^{+}} .
$$

Finally, $\phi_{\ell}^{*}=\phi_{\ell}$ since the boost is along the $z$-axis. Hence

$$
0 \leq \phi_{\ell} \leq 2 \pi \text {. }
$$

\section{B. Differential decay rates}

In this section we derive the fully differential decay rate for $\bar{B} \rightarrow X \pi \ell \bar{\nu}$ employing only the Lorentz and discrete symmetries of QCD, without dynamical considerations. We work

in the $B$ rest frame, and it is convenient to start by using the six independent variables: $q^{2}$, $E_{\ell}, E_{\bar{\nu}}, p_{\pi}^{x}, p_{\pi^{y}}^{y}, p_{\pi}^{z}$. For the fully differential decay rate we have

$$
\frac{d^{6} \Gamma}{d q^{2} d E_{\ell} d E_{\bar{\nu}} d p_{\pi}{ }^{x} d p_{\pi}^{y} d p_{\pi}{ }^{z}}=\frac{\pi^{2}}{(2 \pi)^{6}} \frac{\mathcal{A}}{2 E_{\pi}(2 \pi)^{3}} \theta\left(4 E_{\ell} E_{\bar{\nu}}-q^{2}\right),
$$

where $d^{3} p_{\pi} /\left[2 E_{\pi}(2 \pi)^{3}\right]$ is the phase space for the pion, $E_{\pi}=\sqrt{\vec{p}_{\pi}^{2}+m_{\pi}^{2}}$, and

$$
\begin{aligned}
\mathcal{A} & \equiv \sum_{X} \sum_{\text {l.s. }} \frac{\left\langle\bar{B}\left|H_{W}^{\dagger}\right| X \pi \ell \bar{\nu}\right\rangle\left\langle X \pi \ell \bar{\nu}\left|H_{W}\right| \bar{B}\right\rangle}{2 m_{B}}(2 \pi)^{4} \delta^{4}\left(p_{B}-p_{X \pi}-p_{\ell}-p_{\bar{\nu}}\right) \\
& =16 \pi G_{F}^{2}\left|V_{u b}\right|^{2} L^{\alpha \beta} W_{\alpha \beta} .
\end{aligned}
$$


For the $B$-states we use the relativistic normalization $\langle\bar{B}(\vec{p}) \mid \bar{B}(\vec{q})\rangle=2 E_{B}(2 \pi)^{3} \delta^{3}(\vec{p}-\vec{q})$. In Eq. (23) the effective weak Hamiltonian is

$$
H_{W}=\frac{4 G_{F}}{\sqrt{2}} V_{u b}\left(\bar{u} \gamma_{\mu} P_{L} b\right)\left(\bar{l} \gamma^{\mu} P_{L} \nu_{l}\right)
$$

where $P_{L}=\left(1-\gamma_{5}\right) / 2$, and factoring the leptonic and hadronic parts of the matrix element gives the leptonic tensor $L^{\alpha \beta}$, and the hadronic tensor $W_{\alpha \beta} . L^{\alpha \beta}$ is computed without electroweak radiative corrections, so $L^{\alpha \beta}=\operatorname{Tr}\left[\not p_{\ell} \gamma^{\alpha} P_{L} \not p_{\bar{\nu}} \gamma^{\beta} P_{L}\right]$. The hadronic tensor in the $B$ rest-frame in full QCD is

$$
\begin{aligned}
W_{\mu \nu} & =\frac{1}{2 m_{B}} \sum_{X}(2 \pi)^{3} \delta^{4}\left(p_{B}-p_{X \pi}-q\right)\left\langle\bar{B}\left|J_{\mu}^{u \dagger}(0)\right| X \pi\right\rangle\left\langle X \pi\left|J_{\nu}^{u}(0)\right| \bar{B}\right\rangle \\
& =\frac{1}{4 \pi m_{B}} \int d^{4} x e^{-i q \cdot x} \sum_{X}\left\langle\bar{B}\left|J_{\mu}^{u \dagger}(x)\right| X \pi\right\rangle\left\langle X \pi\left|J_{\nu}^{u}(0)\right| \bar{B}\right\rangle,
\end{aligned}
$$

with the flavor changing weak current $J_{\mu}^{u}=\bar{u} \gamma_{\mu} P_{L} b$. We have $W_{\alpha \beta}=W_{\alpha \beta}\left(p_{\pi}^{\mu}, v^{\mu}, q^{\mu}\right)$ and we will treat this tensor to all orders in $\alpha_{s}$. It can be decomposed using Lorentz invariance, parity, time reversal, and hermiticity into a sum of scalar functions, so

$$
\begin{aligned}
L^{\alpha \beta}= & 2\left(p_{\ell}^{\alpha} p_{\bar{\nu}}^{\beta}+p_{\ell}^{\beta} p_{\bar{\nu}}^{\alpha}-g^{\alpha \beta} p_{\ell} \cdot p_{\bar{\nu}}-i \epsilon^{\alpha \beta \eta \lambda} p_{\ell_{\eta}} p_{\bar{\nu} \lambda}\right), \\
W_{\alpha \beta}= & -g_{\alpha \beta} W_{1}+v_{\alpha} v_{\beta} W_{2}-i \epsilon_{\alpha \beta \mu \nu} v^{\mu} q^{\nu} W_{3}+q_{\alpha} q_{\beta} W_{4}+\left(v_{\alpha} q_{\beta}+v_{\beta} q_{\alpha}\right) W_{5} \\
& +\left(v_{\alpha} p_{\pi_{\beta}}+v_{\beta} p_{\pi_{\alpha}}\right) W_{6}-i \epsilon_{\alpha \beta \mu \nu} p_{\pi}^{\mu} q^{\nu} W_{7}-i \epsilon_{\alpha \beta \mu \nu} v^{\mu} p_{\pi}^{\nu} W_{8}+p_{\pi_{\alpha}} p_{\pi_{\beta}} W_{9} \\
& +\left(p_{\pi_{\alpha}} q_{\beta}+p_{\pi_{\beta}} q_{\alpha}\right) W_{10},
\end{aligned}
$$

with the convention $\epsilon_{0123}=1$. The scalar functions $W_{i}$ depend on the four independent Lorentz invariants $q^{2}, v \cdot q, v \cdot p_{\pi}$ and $p_{\pi} \cdot q$, or four equivalent variables from our desired set,

$$
W_{i}=W_{i}\left(p_{X \pi}^{+}, p_{X \pi}^{-}, p_{\pi}^{+}, p_{\pi}^{-}\right)
$$

To derive Eq. (27) recall that the leptonic variable $q^{\mu}$ equals $m_{B} v^{\mu}-p_{X \pi}^{\mu}$, and can be traded for $p_{X \pi}^{\mu}$. Also recall that $m_{X \pi}^{2}=p_{X \pi}^{-} p_{X \pi}^{+}$. Since the $W_{i}$ do not depend on $\phi_{\ell}$ or $E_{\ell}$ we can (if desired) integrate over these variables without further information about the functional form of the $W_{i}$. In Eq. (26) the $W_{i=1-5}$ are analogs of the tensor coefficients that can appear in the inclusive $\bar{B} \rightarrow X_{u} \ell \bar{\nu}$ decay, but here they induce a more differential decay rate because of the identified pion. The $W_{i=6-10}$ have tensor prefactors involving $p_{\pi}$ and have no analog in the inclusive decay.

Contracting leptonic and hadronic tensors we find

$$
\begin{aligned}
L^{\alpha \beta} W_{\alpha \beta} & =2 q^{2} W_{1}+\left(4 E_{\ell} E_{\bar{\nu}}-q^{2}\right) W_{2}+2 q^{2}\left(E_{\ell}-E_{\bar{\nu}}\right) W_{3} \\
& +\left(4 E_{\ell} p_{\bar{\nu}} \cdot p_{\pi}+4 E_{\bar{\nu}} p_{l} \cdot p_{\pi}-2 E_{\pi} q^{2}\right) W_{6}+2 q^{2}\left(p_{l} \cdot p_{\pi}-p_{\bar{\nu}} \cdot p_{\pi}\right) W_{7} \\
& +4\left(E_{\ell} p_{\bar{\nu}} \cdot p_{\pi}-E_{\bar{\nu}} p_{l} \cdot p_{\pi}\right) W_{8}+\left(4 p_{l} \cdot p_{\pi} p_{\bar{\nu}} \cdot p_{\pi}-m_{\pi}^{2} q^{2}\right) W_{9},
\end{aligned}
$$


where $W_{4,5,10}$ have dropped out since our leptons are massless. In terms of this contraction the fully differential decay rate is

$$
\frac{d^{6} \Gamma}{d q^{2} d E_{\ell} d E_{\bar{\nu}} d p_{\pi}^{x} d p_{\pi}^{y} d p_{\pi}^{z}}=\frac{G_{F}^{2}\left|V_{u b}\right|^{2}}{32 \pi^{6}} \frac{L_{\alpha \beta} W^{\alpha \beta}}{2 E_{\pi}},
$$

where the limits on the kinematic variables are left implicit.

We now want to express Eq. (29) in terms of the coordinates from the previous section: $\left\{p_{X \pi}^{-}, p_{X \pi}^{+}, E_{\ell}, p_{\pi}^{-}, p_{\pi}^{+}, \phi_{\ell}\right\}$. The relations

$$
q^{2}=\left(m_{B}-p_{X \pi}^{-}\right)\left(m_{B}-p_{X \pi}^{+}\right), \quad E_{\bar{\nu}}=m_{B}-E_{\ell}-\left(p_{X \pi}^{-}+p_{X \pi}^{+}\right) / 2,
$$

suffice to convert the $W_{1,2,3}$ terms. For the remaining $W_{i}$ we need expressions for $p_{\ell} \cdot p_{\pi}$, $p_{\bar{\nu}} \cdot p_{\pi}$ and $E_{\pi}$. Recall that $\vec{p}_{X \pi}=-\vec{q}=-\left(\vec{p}_{l}+\vec{p}_{\bar{\nu}}\right)$ is on the $+\hat{z}$-axis, so the leptons are back-to-back in the $\perp$-plane which is transverse to $\hat{z}$. We perform a rotation about the z-axis to bring $\vec{p}_{\pi}$ into the $y$ - $z$ plane with $p_{\pi}^{y} \geq 0$. Then in spherical coordinates $\vec{p}_{\pi}=\left(p_{\pi}^{x}, p_{\pi}^{y}, p_{\pi}^{z}\right)=\left|\vec{p}_{\pi}\right|\left(0, \sin \theta_{\pi}, \cos \theta_{\pi}\right), \vec{p}_{\ell}=E_{\ell}\left(\sin \theta_{\ell} \cos \phi_{\ell}, \sin \theta_{\ell} \sin \phi_{\ell}, \cos \theta_{\ell}\right)$, and $\vec{p}_{\bar{\nu}}=E_{\bar{\nu}}\left(-\sin \theta_{\bar{\nu}} \cos \phi_{\ell},-\sin \theta_{\bar{\nu}} \sin \phi_{\ell}, \cos \theta_{\ell}\right)$, so

$$
\begin{aligned}
p_{\ell} \cdot p_{\pi} & =E_{\ell} E_{\pi}-E_{\ell}\left|\vec{p}_{\pi}\right|\left(\cos \theta_{\ell} \cos \theta_{\pi}+\sin \theta_{\pi} \sin \theta_{\ell} \sin \phi_{\ell}\right), \\
p_{\bar{\nu}} \cdot p_{\pi} & =E_{\bar{\nu}} E_{\pi}-E_{\bar{\nu}}\left|\vec{p}_{\pi}\right|\left(\cos \theta_{\bar{\nu}} \cos \theta_{\pi}-\sin \theta_{\pi} \sin \theta_{\bar{\nu}} \sin \phi_{\ell}\right), \\
E_{\pi} & =\frac{1}{2}\left(p_{\pi}^{-}+p_{\pi}^{+}\right) .
\end{aligned}
$$

Using these expressions the two dot products can be written in terms of the desired variables. First note that

$$
\begin{aligned}
\left|\vec{p}_{\pi}\right| \cos \theta_{\pi} & =\frac{1}{2}\left(p_{\pi}^{-}-p_{\pi}^{+}\right) \\
\left|\vec{p}_{\pi}\right| \sin \theta_{\pi} & =\left(\left|\vec{p}_{\pi}\right|^{2}-\left|\vec{p}_{\pi}\right|^{2} \cos ^{2} \theta_{\pi}\right)^{1 / 2}=\left(p_{\pi}^{-} p_{\pi}^{+}-m_{\pi}^{2}\right)^{1 / 2} .
\end{aligned}
$$

Furthermore, since $\vec{p}_{\ell} \cdot \vec{p}_{\bar{\nu}}=E_{\ell} E_{\bar{\nu}}-q^{2} / 2$, we have $\vec{p}_{\ell} \cdot \vec{q}=-E_{\ell}|\vec{q}| \cos \theta_{\ell}=\vec{p}_{\ell}^{2}+\vec{p}_{\ell} \cdot \vec{p}_{\bar{\nu}}=$ $E_{\ell}^{2}+E_{\ell} E_{\bar{\nu}}-q^{2} / 2$, which, with $|\vec{q}|=\left|\vec{p}_{X \pi}\right|=\left[E_{X \pi}^{2}-m_{X \pi}^{2}\right]^{1 / 2}=\left(p_{X \pi}^{-}-p_{X \pi}^{+}\right) / 2$, implies $E_{\ell} \cos \theta_{\ell}=\left(2 E_{\ell}^{2}+2 E_{\ell} E_{\bar{\nu}}-q^{2}\right) /\left(p_{X \pi}^{+}-p_{X \pi}^{-}\right)$. Hence

$$
\begin{aligned}
E_{\ell} \cos \theta_{\ell} & =\frac{\left(m_{B}-p_{X \pi}^{-}\right)\left(m_{B}-p_{X \pi}^{+}\right)-E_{\ell}\left(2 m_{B}-p_{X \pi}^{-}-p_{X \pi}^{+}\right)}{\left(p_{X \pi}^{-}-p_{X \pi}^{+}\right)}, \\
E_{\bar{\nu}} \cos \theta_{\bar{\nu}} & =\frac{1}{2}\left(p_{X \pi}^{+}-p_{X \pi}^{-}\right)-E_{\ell} \cos \theta_{\ell} .
\end{aligned}
$$

Finally it is useful to note that the equality of the magnitude of the lepton transverse momenta implies $E_{\ell} \sin \theta_{\ell}=E_{\bar{\nu}} \sin \theta_{\bar{\nu}}$.

Together the results in Eqs. (32) and (33) allow us to express $L^{\alpha \beta} W_{\alpha \beta}$ in terms of the six variables $\left\{E_{\ell}, p_{X \pi}^{ \pm}, p_{\pi}^{ \pm}, \phi_{\ell}\right\}$. The only remaining ingredient needed to transform the decay rate to these variables is the Jacobian, which is easily derived by noting that

$$
\frac{d^{3} p_{\pi}}{2 E_{\pi}}=\frac{1}{4} d p_{\pi}^{+} d p_{\pi}^{-} d \phi_{\pi}=\frac{1}{4} d p_{\pi}^{+} d p_{\pi}^{-} d \phi_{\ell} .
$$


For the last equality we used the fact that the pion azimuthal angle becomes equivalent to the lepton azimuthal angle, $d \phi_{\pi} \rightarrow d \phi_{\ell}$, when we rotate the pion momentum into the $y-z$ plane. Although it would be interesting to consider measurements of $\phi_{\ell}$, for our purposes we will integrate over $\phi_{\ell} \in[0,2 \pi]$. Since the $W_{7,8,9}$ prefactors are linear in $p_{\ell} \cdot p_{\pi}$ or $p_{\bar{\nu}} \cdot p_{\pi}$ they have contributions that are either independent of $\phi_{\ell}$ or linear in $\sin \phi_{\ell}$, and the latter terms drop out. In $W_{6}$ the terms linear in $\sin \phi_{\ell}$ do not contribute and a quadratic term averages to $\int_{0}^{2 \pi} d \phi_{\ell} \sin ^{2}\left(\phi_{\ell}\right)=\pi$. All together this gives

$$
\begin{gathered}
\frac{d^{5} \Gamma}{d p_{X \pi}^{+} d p_{X \pi}^{-} d p_{\pi}^{-} d p_{\pi}^{+} d E_{\ell}}=\frac{G_{F}^{2}\left|V_{u b}\right|^{2}}{128 \pi^{5}}\left(\bar{K}_{1} W_{1}+\bar{K}_{2} W_{2}+\bar{K}_{3} W_{3}+\bar{K}_{6} W_{6}+\bar{K}_{7} W_{7}\right. \\
\left.+\bar{K}_{8} W_{8}+\bar{K}_{9} W_{9}\right)
\end{gathered}
$$

multiplied by $\theta\left[\left(p_{X \pi}^{-}+2 E_{\ell}-m_{B}\right)\left(m_{B}-p_{X \pi}^{+}-2 E_{\ell}\right)\right]$ which gives the limits for the $E_{\ell}$ integration, and

$$
\begin{aligned}
\bar{K}_{1} & =2\left(p_{X \pi}^{-}-p_{X \pi}^{+}\right)\left(m_{B}-p_{X \pi}^{-}\right)\left(m_{B}-p_{X \pi}^{+}\right), \\
\bar{K}_{2} & =-\left(p_{X \pi}^{-}-p_{X \pi}^{+}\right)\left(m_{B}-p_{X \pi}^{-}-2 E_{\ell}\right)\left(m_{B}-p_{X \pi}^{+}-2 E_{\ell}\right), \\
\bar{K}_{3} & =\left(p_{X \pi}^{-}-p_{X \pi}^{+}\right)\left(m_{B}-p_{X \pi}^{-}\right)\left(m_{B}-p_{X \pi}^{+}\right)\left(4 E_{\ell}-2 m_{B}+p_{X \pi}^{-}+p_{X \pi}^{+}\right), \\
\bar{K}_{6} & =-2\left(m_{B}-2 E_{\ell}-p_{X \pi}^{-}\right)\left(m_{B}-2 E_{\ell}-p_{X \pi}^{+}\right)\left[m_{B}\left(p_{\pi}^{-}-p_{\pi}^{+}\right)+p_{\pi}^{+} p_{X \pi}^{-}-p_{\pi}^{-} p_{X \pi}^{+}\right], \\
\bar{K}_{7} & =\left(m_{B}-p_{X \pi}^{-}\right)\left(m_{B}-p_{X \pi}^{+}\right)\left(4 E_{\ell}-2 m_{B}+p_{X \pi}^{-}+p_{X \pi}^{+}\right)\left[m_{B}\left(p_{\pi}^{-}-p_{\pi}^{+}\right)+p_{\pi}^{+} p_{X \pi}^{-}-p_{\pi}^{-} p_{X \pi}^{+}\right], \\
\bar{K}_{8} & =\left(p_{\pi}^{-}-p_{\pi}^{+}\right)\left(m_{B}-p_{X \pi}^{-}\right)\left(m_{B}-p_{X \pi}^{+}\right)\left(2 m_{B}-4 E_{\ell}-p_{X \pi}^{-}-p_{X \pi}^{+}\right), \\
\bar{K}_{9} & =\frac{1}{p_{X \pi}^{-}-p_{X \pi}^{+}}\left[\left\{\left(p_{\pi}^{-}-p_{\pi}^{+}\right)\left(m_{B}-p_{X \pi}^{-}\right)\left(m_{B}-p_{X \pi}^{+}\right)-2 E_{\ell}\left[m_{B}\left(p_{\pi}^{-}-p_{\pi}^{+}\right)\right.\right.\right. \\
& \left.\left.+p_{\pi}^{+} p_{X \pi}^{-}-p_{\pi}^{-} p_{X \pi}^{+}\right]\right\}\left\{m_{B}^{2}\left(p_{\pi}^{+}-p_{\pi}^{-}\right)+2 m_{B}\left(p_{\pi}^{-} p_{X \pi}^{+}-p_{\pi}^{+} p_{X \pi}^{-}\right)+p_{\pi}^{+} p_{X \pi}^{-}{ }^{2}-p_{\pi}^{-} p_{X \pi}^{+}{ }^{2}\right. \\
& \left.+2 E_{\ell}\left[m_{B}\left(p_{\pi}^{-}-p_{\pi}^{+}\right)+p_{\pi}^{+} p_{X \pi}^{-}-p_{\pi}^{-} p_{X \pi}^{+}\right]\right\} \\
& \left.+2\left(m_{B}-p_{X \pi}^{-}\right)\left(m_{B}-p_{X \pi}^{+}\right)\left(m_{B}-p_{X \pi}^{-}-2 E_{\ell}\right)\left(m_{B}-p_{X \pi}^{+}-2 E_{\ell}\right)\left(p_{\pi}^{+} p_{\pi}^{-}-m_{\pi}^{2}\right)\right] \\
& -2 m_{\pi}^{2}\left(p_{X \pi}^{-}-p_{X \pi}^{+}\right)\left(m_{B}-p_{X \pi}^{-}\right)\left(m_{B}-p_{X \pi}^{+}\right),
\end{aligned}
$$

where the limits on the hadronic variables are displayed in Eqs. (11) and (19). The $\bar{K}_{i}$ are useful for considering rates where the pion is observed along with a measurement of the charged lepton energy.

Integrating Eq. (35) over the lepton energy $E_{\ell}$, the $W_{3,7,8}$ terms drop out leaving

$$
\frac{d^{4} \Gamma}{d p_{X \pi}^{+} d p_{X \pi}^{-} d p_{\pi}^{-} d p_{\pi}^{+}}=\frac{G_{F}^{2}\left|V_{u b}\right|^{2}}{128 \pi^{5}}\left(K_{1} W_{1}+K_{2} W_{2}+K_{6} W_{6}+K_{9} W_{9}\right)
$$


where

$$
\begin{aligned}
& K_{1}=\left(m_{B}-p_{X \pi}^{-}\right)\left(m_{B}-p_{X \pi}^{+}\right)\left(p_{X \pi}^{-}-p_{X \pi}^{+}\right)^{2}, \\
& K_{2}=\frac{1}{12}\left(p_{X \pi}^{-}-p_{X \pi}^{+}\right)^{4}, \\
& K_{6}=\frac{1}{6}\left(p_{X \pi}^{-}-p_{X \pi}^{+}\right)^{3}\left[m_{B}\left(p_{\pi}^{-}-p_{\pi}^{+}\right)+p_{\pi}^{+} p_{X \pi}^{-}-p_{\pi}^{-} p_{X \pi}^{+}\right], \\
& K_{9}=\frac{1}{12}\left(p_{X \pi}^{-}-p_{X \pi}^{+}\right)^{2}\left\{\left[p_{\pi}^{+}\left(m_{B}-p_{X \pi}^{-}\right)+p_{\pi}^{-}\left(m_{B}-p_{X \pi}^{+}\right)\right]^{2}-4 m_{\pi}^{2}\left(m_{B}-p_{X \pi}^{-}\right)\left(m_{B}-p_{X \pi}^{+}\right)\right\} .
\end{aligned}
$$

No further integrations can be performed in Eq. (37) without first determining the hadronic structure functions $W_{i}\left(p_{X \pi}^{+}, p_{X \pi}^{-}, p_{\pi}^{+}, p_{\pi}^{-}\right)$.

\section{FACTORIZATION WITH A PION FRAGMENTING FROM A JET.}

Using SCET, we derive a leading order factorization theorem for the hadronic structure functions $W_{i}$ appearing in the differential decay rates in section IIB.

We focus on the region of phase space with endpoint jet-like kinematics where $p_{X \pi}^{+} \ll p_{X \pi}^{-}$, and with an energetic pion produced by fragmentation with $p_{\pi}^{+} \ll p_{\pi}^{-}$. It is assumed that suitable phase-space cuts are applied to subtract the $b \rightarrow c$ background, which phenomenologically is responsible for the importance of this kinematic endpoint region. This issue is explored in a separate publication [39]. With $p_{\pi}^{+} \leq p_{\pi}^{-}$the boundaries for $p_{\pi}^{+}$and $p_{\pi}^{-}$in Eq. (19) become:

$$
\frac{m_{\pi}^{2}}{p_{X \pi}^{+}} \leq p_{\pi}^{-} \leq p_{X \pi}^{-}, \quad \frac{m_{\pi}^{2}}{p_{\pi}^{-}} \leq p_{\pi}^{+} \leq \min \left\{p_{\pi}^{-}, p_{X \pi}^{+}\left(1-\frac{p_{\pi}^{-}}{p_{X \pi}^{-}}\right)+\frac{m_{\pi}^{2}}{p_{X \pi}^{-}}\right\},
$$

or, reversing the order as in Eq. (20),

$$
\frac{m_{\pi}^{2}}{p_{X \pi}^{-}} \leq p_{\pi}^{+} \leq p_{X \pi}^{+}, \quad \max \left\{\frac{m_{\pi}^{2}}{p_{\pi}^{+}}, p_{\pi}^{+}\right\} \leq p_{\pi}^{-} \leq p_{X \pi}^{-}\left(1-\frac{p_{\pi}^{+}}{p_{X \pi}^{+}}\right)+\frac{m_{\pi}^{2}}{p_{X \pi}^{+}} .
$$

For jet-like final hadronic states, the relevant power counting is $E_{X \pi} \sim m_{b}, m_{b}^{2} \gg$ $m_{X}^{2} \gtrsim m_{b} \Lambda_{\mathrm{QCD}}$, and $p_{\pi}^{-} \sim m_{b}$. If we decompose the momentum of the remainder of the collinear jet after the emission of the pion as $p_{X}^{\mu}=\left(p_{X}^{+}, p_{X}^{-}, p_{X}^{\perp}\right)$, then it scales as

$p_{X}^{\mu} \sim\left(\Lambda_{\mathrm{QCD}}, m_{b}, \sqrt{m_{b} \Lambda_{\mathrm{QCD}}}\right)=m_{b}\left(\lambda^{2}, 1, \lambda\right)$ where $\lambda \sim \sqrt{\Lambda_{\mathrm{QCD}} / m_{b}}$ is the SCET expansion parameter (which can be defined as $\lambda^{2}=m_{X \pi}^{2} / m_{B}^{2}$ for our process). The total hadronic momentum $p_{X \pi}^{\mu}$ is also collinear and scales the same way as $p_{X}^{\mu}$. We will start by considering the $(X \pi)$ system as a combined collinear jet, to be factored from the hard dynamics at the scale $m_{b}$, and the soft dynamics responsible for the binding of quarks in the $B$ meson. This part of the computation can be carried out in SCET $\mathrm{I}$ with collinear and ultrasoft (usoft) degrees of freedom. The energetic pion fragments from the jet and has a collinear scaling $p_{\pi}^{\mu} \sim\left(\Lambda_{\mathrm{QCD}}^{2} / m_{b}, m_{b}, \Lambda_{\mathrm{QCD}}\right)$ with much smaller invariant mass $p_{\pi}^{2} \ll m_{X \pi}^{2}$. The factorization for this second fragmentation step can be carried out by a $\mathrm{SCET}_{\mathrm{I}}$ to $\mathrm{SCET}_{\mathrm{II}}$ matching computation [40]. 
We shall now consider Eq. (25) at leading order in SCET to derive factorized expressions for the scalar structure functions $W_{i}$ in the fragmentation region. We work in a frame where $q_{\perp}=0$, which will induce a vanishing $\perp$-label momentum for the light quark field in the partonic subprocess and the $X \pi$ system. Since the $X \pi$ system is collinear, it is convenient to decompose momenta as $p^{\mu}=p_{l}^{\mu}+p_{r}^{\mu}$ where we have label momenta $p_{l}^{-} \sim \lambda^{0}, p_{l}^{\perp} \sim \lambda$, and residual momenta $p_{r}^{\mu} \sim \lambda^{2}$. In SCET the pion phase space integral can be written as

$$
\int \frac{d^{3} p_{\pi}}{2 E_{\pi}} W\left(\vec{p}_{\pi}\right)=\int \frac{d p_{\pi}^{-} d^{2} p_{\pi}^{\perp}}{2 p_{\pi}^{-}} W\left(p_{\pi}^{-}, p_{\pi}^{\perp}\right)=\sum_{p_{\pi l}^{-}} \sum_{p_{\pi l}^{\perp}} \int \frac{d p_{\pi r}^{-} d^{2} p_{\pi r}^{\perp}}{2 p_{\pi l}^{-}} W\left(p_{\pi l}^{-}, p_{\pi l}^{\perp}\right)
$$

The same holds for the variables $p_{X}^{-}$and $p_{X}^{\perp}$. Thus for all the $W=W_{i}$, we can treat $p_{X \pi}^{-}$, $p_{X \pi}^{\perp}, p_{\pi}^{-}$, and $p_{\pi}^{\perp}$ as discrete label momenta. At the end these variables are restored to continuous variables using Eq. (41) and the analogs for phase space integrations over the $X \pi$ variables.

Matching the heavy-to-light QCD current onto SCET operators at a scale of order $m_{b}$, at leading order one obtains [9]

$$
J_{u}^{\nu}(x)=e^{i \mathcal{P} \cdot x-i m_{b} v \cdot x} \sum_{j=1}^{3} \sum_{\omega} C_{j}(\omega) J_{u j}^{\nu(0)}(\omega) .
$$

Here $\mathcal{P}^{\mu}=n^{\mu} \overline{\mathcal{P}} / 2+\mathcal{P}_{\perp}^{\mu}$ where $\overline{\mathcal{P}}$ and $\mathcal{P}_{\perp}$ are the $\mathcal{O}\left(\lambda^{0}\right)$ and $\mathcal{O}(\lambda)$ label momentum operators [10]. The leading order SCET current becomes

$$
J_{u j}^{\nu(0)}(\omega)=\bar{\chi}_{n, \omega} \Gamma_{j}^{\nu} \mathcal{H}_{v}
$$

In this expression, $\bar{\chi}_{n, \omega} \equiv\left(\bar{\xi}_{n} W_{n}\right) \delta_{\omega} \overline{\mathcal{P}}^{\dagger}$, where $\xi_{n}$ is the $n$-collinear light $u$-quark field. The collinear Wilson line is defined as [10]

$$
W_{n}=\sum_{\text {perms }} \exp \left(-\frac{g}{\overline{\mathcal{P}}} \bar{n} \cdot A_{n}(x)\right)
$$

with collinear gluons $A_{n}$. Also $\mathcal{H}_{v} \equiv Y^{\dagger} h_{v}$, where $h_{v}$ is the ultrasoft heavy quark effective theory field, and $Y(x)=P \exp \left(i g \int_{-\infty}^{0} d s n \cdot A_{u s}(n s+x)\right)$ is a Wilson line built out of ultrasoft gauge fields, which results from decoupling the ultrasoft gluons from the leadingorder collinear Lagrangian [7]. For the leading-order Dirac structures we use the basis [41, 42]

$$
\Gamma_{1}^{\nu}=P_{R} \gamma^{\nu}, \quad \Gamma_{2}^{\nu}=P_{R} v^{\nu}, \quad \Gamma_{3}^{\nu}=P_{R} \frac{n^{\nu}}{n \cdot v},
$$

where $P_{R}=\left(1+\gamma_{5}\right) / 2$. Expressions of the one-loop Wilson coefficients $C_{j}$ can be found in Ref. [7] and the two-loop coefficients were obtained recently by several groups in Refs. [4346]. 
For our derivation of the leading-order factorization formula in $\mathrm{SCET}_{\mathrm{I}}$ we follow the steps in Ref. [34], except that we will write out the dependence on $\mathcal{P}_{\perp}$ explicitly. When the current in Eq. (42) is inserted in Eq. (25) we have the phase

$$
\int \mathrm{d}^{4} x e^{-i q \cdot x} e^{i \mathcal{P} \cdot x-i m_{b} v \cdot x}=\delta_{\overline{\mathcal{P}}, \bar{n} \cdot p} \delta_{\mathcal{P}_{\perp}, 0} \int d^{4} x e^{-i r \cdot x},
$$

where $\bar{n} \cdot p=m_{b}-q^{-}$and we used the fact that $q_{\perp}=0$. Eq. (46) leaves discrete $\delta$ 's that fix the label momenta, and a $d^{4} x$ integration that only involves the residual momentum $r^{\mu}=\bar{n}^{\mu} r^{+} / 2$ with $r^{+}=m_{b}-q^{+} \sim \lambda^{2}$. Using the normalization convention in Heavy Quark Effective Theory, $\left\langle\bar{B}_{v}\left(\vec{k}^{\prime}\right) \mid \bar{B}_{v}(\vec{k})\right\rangle=2 v^{0}(2 \pi)^{3} \delta^{3}\left(\vec{k}-\vec{k}^{\prime}\right)$, the leading order expression for Eq. (25) in SCET becomes

$$
\begin{aligned}
W_{\mu \nu}^{(0)} & =\frac{1}{4 \pi} \int d^{4} x e^{-i r \cdot x} \sum_{j, j^{\prime}=1}^{3} \sum_{\omega, \omega^{\prime}} C_{j^{\prime}}\left(\omega^{\prime}\right) C_{j}(\omega) \delta_{\omega^{\prime}, \bar{n} \cdot p} \\
& \times \sum_{X}\left\langle\bar{B}_{v}\left|\left[\overline{\mathcal{H}}_{v} \bar{\Gamma}_{j^{\prime} \mu} \chi_{n, \omega^{\prime}, 0_{\perp}}\right](x)\right| X \pi\right\rangle\left\langle X \pi\left|\left[\bar{\chi}_{n, \omega} \Gamma_{j \nu} \mathcal{H}_{v}\right](0)\right| \bar{B}_{v}\right\rangle
\end{aligned}
$$

where $\chi_{n, \omega^{\prime}, 0_{\perp}} \equiv \delta_{\omega, \overline{\mathcal{P}}} \delta_{0, \mathcal{P}_{\perp}}\left(W_{n}^{\dagger} \xi_{n}\right)$. Here $\bar{\Gamma}_{j^{\prime}, \mu}=\gamma_{0} \Gamma_{j^{\prime} \mu}^{\dagger} \gamma_{0}$ and $\bar{n} \cdot p=p^{-}$is the large momentum of the energetic quark producing the jet. Grouping ultrasoft and collinear fields by a Fierz transformation, we have

$$
\begin{aligned}
{\left[\overline{\mathcal{H}}_{v} \bar{\Gamma}_{j^{\prime} \mu} \chi_{n, \omega^{\prime}, 0_{\perp}}\right](x)\left[\bar{\chi}_{n, \omega} \Gamma_{j \nu} \mathcal{H}_{v}\right](0) } & =(-1)\left[\overline{\mathcal{H}}_{v}(x) \bar{\Gamma}_{j^{\prime} \mu} \frac{\not h}{2} \Gamma_{j \nu} \mathcal{H}_{v}(0)\right]\left[\bar{\chi}_{n, \omega}(0) \frac{\not h}{4 N_{c}} \chi_{n, \omega^{\prime}, 0_{\perp}}(x)\right] \\
& +\ldots,
\end{aligned}
$$

where one should keep in mind that the $\left\langle B_{v}|\cdots| B_{v}\right\rangle$ states will surround the $\mathcal{H}_{v}$ bilinear, and the $\langle 0|\cdots| X \pi\rangle\langle X \pi|\cdots| 0\rangle$ states split the $\chi_{n, \omega}$ field bilinear into two parts. The ellipses in Eq. (48) denote Dirac and color structures that vanish either because they involve an octet matrix $T^{a}$ between the color singlet $\left|\bar{B}_{v}\right\rangle$ states, or by parity, or because the only available vector for the $\left\langle B_{v}|\cdots| B_{v}\right\rangle$ matrix element is $v^{\mu}$, and $v_{\perp}^{\mu}=0$. The form of the collinear product of matrix elements is pictured in Fig. 2, and the most general allowed parameterization is

$$
\begin{aligned}
& \frac{1}{4 N_{c}} \operatorname{Tr} \sum_{X} \not h\left\langle 0\left|\chi_{n, \omega^{\prime}, 0_{\perp}}(x)\right| X \pi\right\rangle\left\langle X \pi\left|\bar{\chi}_{n, \omega}(0)\right| 0\right\rangle= \\
& \quad=2 \delta_{\omega, \omega^{\prime}} \delta\left(x^{+}\right) \delta^{2}\left(x_{\perp}\right) \omega \int \frac{d k^{+}}{2 \pi} e^{-i k^{+} x^{-} / 2} \overline{\mathcal{G}}_{u}^{\pi}\left(k^{+} \omega, \frac{p_{\pi}^{-}}{\omega}, p_{\pi}^{+} p_{\pi}^{-}\right),
\end{aligned}
$$

where the trace is over color and Dirac indices and Eq. (47) implies that $\omega=\bar{n} \cdot p$. The first $\delta$-function in Eq. (49) stems from label momentum conservation and the remaining ones from the fact that the leading collinear Lagrangian contains only the $n \cdot \partial$ derivative. The arguments of $\overline{\mathcal{G}}_{u}^{\pi}$ are constrained by RPI-III invariance [47], which requires products of plus-momenta and minus-momenta, or ratios of minus- (or plus-) momenta. (For our 


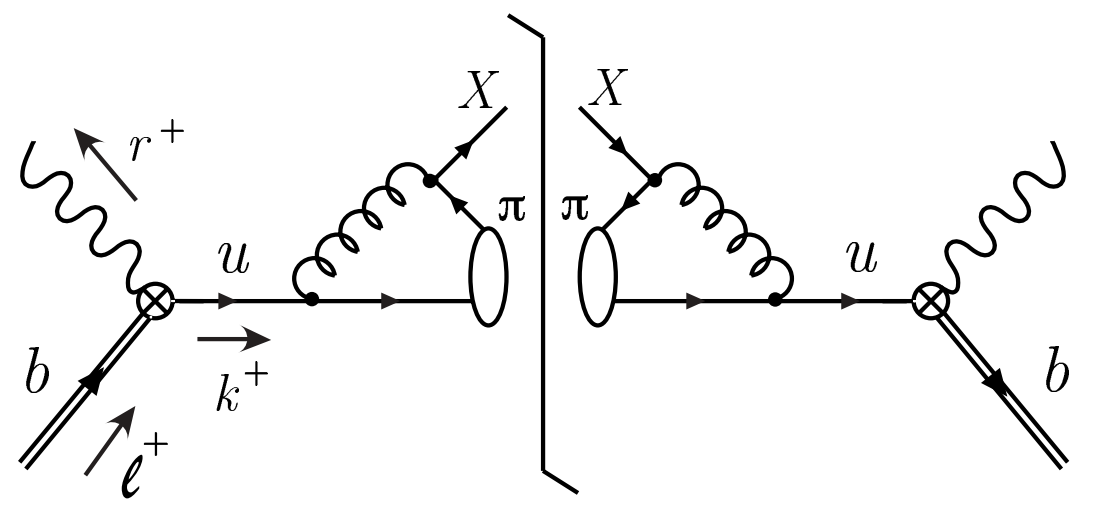

FIG. 2: Sketch of the hadronic fragmentation process for $\bar{B} \rightarrow X \pi \ell \bar{\nu}$.

case RPI-III is equivalent to invariance under boosts along the $\hat{z}$ jet axis.) The arguments of $\overline{\mathcal{G}}_{u}^{\pi}$ are also constrained by plus-momentum conservation. The light-cone variable $k^{+}$is the plus-momentum of the up-quark initiating the $X \pi$ production, and at the interaction vertex is related to the residual (soft) plus-momentum $\ell^{+}$of the $b$-quark in the $B$-meson by $k^{+}=\ell^{+}-r^{+}$, as shown in Fig. 2, ${ }^{2}$ The large label partonic momentum $\bar{n} \cdot p$ also is fixed in terms of kinematic variables:

$$
\bar{n} \cdot p=m_{b}-\bar{n} \cdot q=m_{b}-m_{B}+\bar{n} \cdot p_{X \pi}=\bar{n} \cdot p_{X \pi}-\bar{\Lambda}+\mathcal{O}\left(\frac{\Lambda_{\mathrm{QCD}}^{2}}{m_{b}}\right) .
$$

Since $\bar{\Lambda}=\mathcal{O}\left(\Lambda_{\mathrm{QCD}}\right)$, the ratio $p_{\pi}^{-} / p_{X \pi}^{-}$is identified to leading order with $p_{\pi}^{-} / \omega=p_{\pi}^{-} / p^{-} \equiv z$, the fragmentation variable in Eq. (7).

Since $p_{\pi}^{+} p_{\pi}^{-}=m_{\pi}^{2}+\vec{p}_{\pi}^{\perp 2}, \overline{\mathcal{G}}_{u}^{\pi}$ depends on $\left|\vec{p}_{\pi}^{\perp}\right|$, which is non-vanishing with our choice of coordinates (the pion has $\vec{p}_{\pi}^{\perp}$ and the $\perp$-momentum of $X$ is $-\vec{p}_{\pi}^{\perp}$ ). In general it is the relative $\perp$-momentum between $\pi, X$, and the $B$ that can not be transformed to zero. Later we will integrate over $\left|p_{\pi}^{\perp}\right|$ or equivalently $p_{\pi}^{+}$, and study

$$
\mathcal{G}_{u}^{\pi}\left(k^{+} \omega, z, \mu\right) \equiv \omega \int d p_{\pi}^{+} \overline{\mathcal{G}}_{u}^{\pi}\left(k^{+} \omega, z, p_{\pi}^{+} p_{\pi}^{-}, \mu\right)
$$

which occurs in

$$
\begin{aligned}
& \frac{1}{4 N_{c}} \operatorname{Tr} \int d p_{\pi}^{+} \sum_{X} \not{\not h}\left\langle 0\left|\chi_{n, \omega^{\prime}, 0_{\perp}}(x)\right| X \pi\right\rangle\left\langle X \pi\left|\bar{\chi}_{n, \omega}(0)\right| 0\right\rangle= \\
& \quad=2 \delta_{\omega, \omega^{\prime}} \delta\left(x^{+}\right) \delta^{2}\left(x_{\perp}\right) \int \frac{d k^{+}}{2 \pi} e^{-i k^{+} x^{-} / 2} \mathcal{G}_{u}^{\pi}\left(k^{+} \omega, \frac{p_{\pi}^{-}}{\omega}\right) .
\end{aligned}
$$

The fragmenting jet function $\overline{\mathcal{G}}_{u}^{\pi}$ defined in Eq. (49) describes the properties of a final state that is collimated in the $\vec{n}$-direction and consists of a up-quark initiated jet from which

\footnotetext{
${ }^{2}$ Strictly speaking the result $k^{+}=\ell^{+}-r^{+}$also encodes the presence of Wilson lines in defining these momenta, which ensure gauge invariance.
} 
a pion fragments. Unlike the standard unpolarized parton fragmentation function $D_{i}^{\pi}(z)$, $\overline{\mathcal{G}}_{u}^{\pi}\left(s, z, p_{\pi}^{+} p_{\pi}^{-}\right)$carries information about the invariant mass $s$ of the fragmenting jet and the direction of the fragmenting pion through $p_{\pi}^{+} p_{\pi}^{-}$. The matrix elements in Eqs. (49) and (52) are similar to the collinear matrix element defining the jet function $J(s)$ that appears in $\bar{B} \rightarrow X_{u} \ell \bar{\nu}$ and $\bar{B} \rightarrow X_{s} \gamma$. The jet function can be written as [4]

$$
\frac{1}{4 N_{c}} \operatorname{Tr} \sum_{X_{u}}\left\langle 0\left|\not h \chi_{n}(x)\right| X_{u}\right\rangle\left\langle X_{u}\left|\bar{\chi}_{n, \omega, 0_{\perp}}(0)\right| 0\right\rangle=\delta\left(x^{+}\right) \delta^{2}\left(x_{\perp}\right) \omega \int d k^{+} e^{-i k^{+} x^{-} / 2} J_{u}\left(\omega k^{+}\right) .
$$

$J_{u}(s)$ depends on the product $s=k^{+} p_{X_{u}}^{-}$, which is analogous to the first argument in $\overline{\mathcal{G}}_{u}^{\pi}$.

The sum in Eq. (53) extends over states with invariant mass up to $m_{X_{u}}^{2} \sim m_{b} \Lambda_{\mathrm{QCD}}$, which are complete in the endpoint region. Hence one can write $J$ as the imaginary part (or discontinuity) of a time-ordered product:

$$
J_{u}\left(k^{+} \omega\right)=-\frac{1}{\pi \omega} \operatorname{Im} \int d^{4} x e^{i k \cdot x} i\left\langle 0\left|\mathrm{~T} \bar{\chi}_{n, \omega, 0_{\perp}}(0) \frac{\not h}{4 N_{c}} \chi_{n}(x)\right| 0\right\rangle,
$$

which is perturbatively calculable with

$$
J_{u}(s)=\delta(s)+\mathcal{O}\left(\alpha_{s}\right)
$$

On the other hand, $\overline{\mathcal{G}}_{u}^{\pi}$ involves a pion state, and therefore contains both perturbative and non-perturbative parts. A simple discontinuity formula like Eq.(54) does not exist for $\overline{\mathcal{G}}_{u}^{\pi}$ with the states $\{|X \pi\rangle\}$.

Combining Eqs. (47) and (49) the hadronic tensor at leading order becomes

$$
\begin{aligned}
W_{\mu \nu} & =\frac{1}{4 \pi} \int d x^{-} e^{-i r^{+} x^{-} / 2} \sum_{j, j^{\prime}=1}^{3} C_{j^{\prime}}\left(m_{b}, p_{X \pi}^{-}\right) C_{j}\left(m_{b}, p_{X \pi}^{-}\right) \operatorname{Tr}\left[\frac{P_{v}}{2} \bar{\Gamma}_{j^{\prime} \mu}^{(0)} \frac{\not h}{2} \Gamma_{j \nu}^{(0)}\right] \\
& \times p_{X \pi}^{-} \int \frac{d k^{+}}{2 \pi} e^{-i k^{+} x^{-} / 2} \overline{\mathcal{G}}_{u}^{\pi}\left(k^{+} p_{X \pi}^{-}, \frac{p_{\pi}^{-}}{p_{X \pi}^{-}}, p_{\pi}^{+} p_{\pi}^{-}\right)\left\langle\bar{B}_{v}\left|\bar{h}_{v}(\tilde{x}) Y(\tilde{x}, 0) h_{v}(0)\right| \bar{B}_{v}\right\rangle \\
& \times\left[1+\mathcal{O}\left(\frac{\Lambda_{\mathrm{QCD}}^{2}}{m_{X \pi}^{2}}\right)\right],
\end{aligned}
$$

where

$$
P_{v}=\frac{1+\psi}{2}, \quad Y(x, y)=Y(x) Y^{\dagger}(y), \quad \tilde{x}^{\mu}=\bar{n} \cdot x n^{\mu} / 2 .
$$

The matrix element of the bilocal operator in Eq. (56) defines the leading-order shape function [48, 49]

$$
f\left(l^{+}\right)=\frac{1}{2} \int \frac{d x^{-}}{4 \pi} e^{-i x^{-} l^{+} / 2}\left\langle\bar{B}_{v}\left|\bar{h}_{v}(\tilde{x}) Y(\tilde{x}, 0) h_{v}(0)\right| \bar{B}_{v}\right\rangle=\frac{1}{2}\left\langle\bar{B}_{v}\left|\bar{h}_{v} \delta\left(l^{+}-i n \cdot D\right) h_{v}\right| \bar{B}_{v}\right\rangle
$$

with $l^{+}=r^{+}+k^{+}$for Eq. (56) $)$. In the limit $m_{b} \rightarrow \infty$, the support of $f$ is $(-\infty, \bar{\Lambda}]$. The shape function accounts for non-perturbative soft dynamics in the $B$-meson. Defining projectors 
$P_{i}^{\mu \nu}$ such that $W_{i}=W_{\mu \nu} P_{i}^{\mu \nu}$, we obtain the leading power result

$$
W_{i}^{(0)}=\frac{h_{i}}{\pi} p_{X \pi}^{-} \int_{0}^{\bar{\Lambda}-r^{+}} d k^{+} \overline{\mathcal{G}}_{u}^{\pi}\left(k^{+} p_{X \pi}^{-}, \frac{p_{\pi}^{-}}{p_{X \pi}^{-}}, p_{\pi}^{+} p_{\pi}^{-}, \mu\right) f\left(k^{+}+r^{+}, \mu\right),
$$

where we show explicitly the dependence on $\mu$, the renormalization scale. Here $h_{i}=$ $h_{i}\left(m_{b}, p_{X \pi}^{-}, p_{X \pi}^{+}, \mu\right)$ where the dependence on $p_{X \pi}^{+}$is entirely from contractions in the tensors, while that on $m_{b}, p_{X \pi}^{-}, \mu$ comes also from loops. In terms of the Wilson coefficients,

$$
h_{i}=\sum_{j, j^{\prime}=1}^{3} C_{j^{\prime}}\left(m_{b}, p_{X \pi}^{-}, \mu\right) C_{j}\left(m_{b}, p_{X \pi}^{-}, \mu\right) \operatorname{Tr}\left[\frac{P_{v}}{2} \bar{\Gamma}_{j^{\prime} \mu}^{(0)} \frac{\not h}{2} \Gamma_{j \nu}^{(0)}\right] P_{i}^{\mu \nu} .
$$

The projectors $P_{i}^{\mu \nu}$ relevant for the differential decay rates in Eqs. (35) and (37) have the following structure:

$$
\begin{aligned}
P_{i}^{\mu \nu}= & A_{i} g^{\mu \nu}+B_{i} v^{\mu} v^{\nu}+C_{i} q^{\mu} q^{\nu}+D_{i}\left(v^{\mu} q^{\nu}+v^{\nu} q^{\mu}\right)+E_{i} p_{\pi}^{\mu} p_{\pi}^{\nu}+F_{i}\left(v^{\mu} p_{\pi}^{\nu}+v^{\nu} p_{\pi}^{\mu}\right)+ \\
& +G_{i}\left(p_{\pi}^{\mu} q^{\nu}+p_{\pi}^{\nu} q^{\mu}\right)+H_{i} i \epsilon^{\mu \nu \alpha \beta} v_{\alpha} q_{\beta}+I_{i} i \epsilon^{\mu \nu \alpha \beta} p_{\alpha}^{\pi} q_{\beta}+L_{i} i \epsilon^{\mu \nu \alpha \beta} v_{\alpha} p_{\beta}^{\pi},
\end{aligned}
$$

where the coefficients $A_{i} \ldots L_{i}$ are functions of $p_{X \pi}^{+}, p_{X \pi}^{-}, p_{\pi}^{+}, p_{\pi}^{-}$and $m_{B}$ that are straightforward to determine by inverting the result for $W_{\alpha \beta}$ in Eq. (26).

In terms of hadronic variables, Eq. (59) becomes

$$
\begin{aligned}
W_{i}^{(0)} & =\frac{h_{i}}{\pi} p_{X \pi}^{-} \int_{0}^{p_{X \pi}^{+}} d k^{+} \overline{\mathcal{G}}_{u}^{\pi}\left(k^{+} p_{X \pi}^{-}, \frac{p_{\pi}^{-}}{p_{X \pi}^{-}}, p_{\pi}^{+} p_{\pi}^{-}, \mu\right) f\left(k^{+}+\bar{\Lambda}-p_{X \pi}^{+}, \mu\right) \\
& =\frac{h_{i}}{\pi} p_{X \pi}^{-} \int_{0}^{p_{X \pi}^{+}} d k^{+} \overline{\mathcal{G}}_{u}^{\pi}\left(k^{+} p_{X \pi}^{-}, \frac{p_{\pi}^{-}}{p_{X \pi}^{-}}, p_{\pi}^{+} p_{\pi}^{-}, \mu\right) S\left(p_{X \pi}^{+}-k^{+}, \mu\right) \\
& =\frac{h_{i}}{\pi} p_{X \pi}^{-} \int_{0}^{p_{X \pi}^{+}} d k^{\prime+} \overline{\mathcal{G}}_{u}^{\pi}\left(p_{X \pi}^{-}\left(p_{X \pi}^{+}-k^{++}\right), \frac{p_{\pi}^{-}}{p_{X \pi}^{-}}, p_{\pi}^{+} p_{\pi}^{-}, \mu\right) S\left(k^{+}, \mu\right)
\end{aligned}
$$

where $S(p) \equiv f(\bar{\Lambda}-p)$ has support for $p \geq 0$. The convolution variable $k^{+} \equiv p_{X \pi}^{+}-k^{+}$ represents the plus-momentum of the light-degrees of freedom (soft gluons, quarks, and antiquarks) in the $B$-meson, and $p_{X \pi}^{-}\left(p_{X \pi}^{+}-k^{\prime+}\right)$ is the invariant mass of collinear particles in the $u$-quark jet including the fragmentation pion.

Evaluating the traces in Eq. (60), we derive from Eq. (35) the following factorization formula for the endpoint fivefold differential decay rate:

$$
\begin{aligned}
\frac{d^{5} \Gamma}{d p_{X \pi}^{+} d p_{X \pi}^{-} d p_{\pi}^{-} d p_{\pi}^{+} d E_{\ell}} & =3 \Gamma_{0} \bar{H}\left(m_{B}, p_{X \pi}^{-}, p_{X \pi}^{+}, E_{\ell}, \mu\right) p_{X \pi}^{-} \\
& \times \int_{0}^{p_{X \pi}^{+}} d k^{\prime+} \overline{\mathcal{G}}_{u}^{\pi}\left(p_{X \pi}^{-}\left(p_{X \pi}^{+}-k^{\prime+}\right), \frac{p_{\pi}^{-}}{p_{X \pi}^{-}}, p_{\pi}^{+} p_{\pi}^{-}, \mu\right) S\left(k^{\prime+}, \mu\right),
\end{aligned}
$$


with $\Gamma_{0} \equiv G_{F}^{2}\left|V_{u b}\right|^{2} /\left(1536 \pi^{6}\right)$ and

$$
\begin{gathered}
\bar{H}\left(m_{B}, p_{X \pi}^{-}, p_{X \pi}^{+}, E_{\ell}, \mu\right)=4\left(m_{B}-p_{X \pi}^{-}-2 E_{\ell}\right)\left\{\left(m_{B}-p_{X \pi}^{+}\right)\left(2 E_{\ell}-2 m_{B}+p_{X \pi}^{-}+p_{X \pi}^{+}\right) C_{1}^{2}\right. \\
+\left(2 E_{\ell}-m_{B}+p_{X \pi}^{+}\right)\left[\left(m_{B}-p_{X \pi}^{+}\right) C_{1} C_{2}+\left(p_{X \pi}^{-}-p_{X \pi}^{+}\right) \frac{C_{2}^{2}}{4}\right. \\
\left.\left.+2 \frac{\left(m_{B}-p_{X \pi}^{+}\right)^{2}}{p_{X \pi}^{-}-p_{X \pi}^{+}} C_{1} C_{3}+\left(m_{B}-p_{X \pi}^{+}\right) C_{2} C_{3}+\frac{\left(m_{B}-p_{X \pi}^{+}\right)^{2}}{p_{X \pi}^{-}-p_{X \pi}^{+}} C_{3}^{2}\right]\right\}
\end{gathered}
$$

The $p_{X \pi^{-}}^{+}$and $E_{\ell^{-}}$dependence in this expression comes solely from contraction of the leptonic and hadronic tensors. The renormalized Wilson coefficients which encode hard loop corrections are functions $C_{i}=C_{i}\left(m_{b}, p_{X \pi}^{-}, \mu\right)$.

For the decay rate in Eq. (37) which integrates over the lepton energy we obtain:

$$
\begin{aligned}
\frac{d^{4} \Gamma}{d p_{X \pi}^{+} d p_{X \pi}^{-} d p_{\pi}^{-} d p_{\pi}^{+}}= & \Gamma_{0} H\left(m_{B}, p_{X \pi}^{-}, p_{X \pi}^{+}, \mu\right) p_{X \pi}^{-} \\
& \times \int_{0}^{p_{X \pi}^{+}} d k^{\prime+} \overline{\mathcal{G}}_{u}^{\pi}\left(p_{X \pi}^{-}\left(p_{X \pi}^{+}-k^{+}\right), \frac{p_{\pi}^{-}}{p_{X \pi}^{-}}, p_{\pi}^{+} p_{\pi}^{-}, \mu\right) S\left(k^{\prime+}, \mu\right),
\end{aligned}
$$

where

$$
\begin{aligned}
H\left(m_{B}, p_{X \pi}^{-}, p_{X \pi}^{+}, \mu\right)= & \left(p_{X \pi}^{-}-p_{X \pi}^{+}\right)^{2}\left[\left(m_{B}-p_{X \pi}^{+}\right)\left(3 m_{B}-2 p_{X \pi}^{-}-p_{X \pi}^{+}\right) C_{1}^{2}\right. \\
& +\left(m_{B}-p_{X \pi}^{+}\right)\left(p_{X \pi}^{-}-p_{X \pi}^{+}\right) C_{1} C_{2}+\left(p_{X \pi}^{-}-p_{X \pi}^{+}\right)^{2} \frac{C_{2}^{2}}{4} \\
& +2\left(m_{B}-p_{X \pi}^{+}\right)^{2} C_{1} C_{3}+\left(m_{B}-p_{X \pi}^{+}\right)\left(p_{X \pi}^{-}-p_{X \pi}^{+}\right) C_{2} C_{3} \\
& \left.+\left(m_{B}-p_{X \pi}^{+}\right)^{2} C_{3}^{2}\right] .
\end{aligned}
$$

The functions $\bar{H}$ and $H$ encode contributions from hard scales, and from the kinematic contraction of tensor coefficients. In the phase space region where $p_{X \pi}^{+} \sim \Lambda_{\mathrm{QCD}}$, at leading order in the SCET power counting,

$$
H\left(m_{B}, p_{X \pi}^{-}, p_{X \pi}^{+}, \mu\right)=H\left(m_{b}, p^{-}, 0, \mu\right)
$$

The same considerations apply to the function $H\left(m_{B}, p_{X \pi}^{-}, p_{X \pi}^{+}, E_{\ell}, \mu\right)$ in Eq.(63). Most often it is useful to treat the $p_{X \pi}^{+}$dependence from the tensor contractions exactly, without expanding $p_{X \pi}^{+} \ll p_{X \pi}^{-}$, since at lowest order in the perturbative corrections this allows [37] the endpoint jet-like factorization theorem to agree with results derived in the more inclusive situation where $m_{X_{u}}^{2} \sim m_{b}^{2}$.

For the purpose of comparison with phenomenology, it is appropriate to derive the expression of the decay rate which is doubly differential in the jet invariant mass and in the fraction $z$ of large momentum components. Let us first integrate Eq. (65) over $p_{\pi}^{+}$. At leading order we can set $m_{\pi}=0$, since $m_{\pi}^{2}=\mathcal{O}\left(\lambda^{4}\right)$. Therefore, in the chiral limit, $p_{\pi}^{+} \geq 0$ 
from Eq. (39) . Since the pion fragments from the jet, the maximum value of $p_{\pi}^{+}$is $p_{X \pi}^{+}-k^{\prime+}$. Hence we can write

$$
\frac{d^{3} \Gamma}{d p_{X \pi}^{+} d p_{X \pi}^{-} d p_{\pi}^{-}}=\Gamma_{0} H\left(m_{B}, p_{X \pi}^{-}, p_{X \pi}^{+}, \mu\right) \int_{0}^{p_{X \pi}^{+}} d k^{+} \mathcal{G}_{u}^{\pi}\left(k^{+} p_{X \pi}^{-}, z, \mu\right) S\left(p_{X \pi}^{+}-k^{+}, \mu\right)
$$

where $\mathcal{G}_{u}^{\pi}$ is defined in Eq. (51). By integrating further:

$$
\frac{d^{2} \Gamma}{d m_{X \pi}^{2} d z}=\left.\left.\int_{m_{X \pi}^{2} / m_{B}}^{m_{X \pi}} d p_{X \pi}^{+} \frac{m_{X \pi}^{2}}{\left(p_{X \pi}^{+}\right)^{2}} \frac{d^{3} \Gamma}{d p_{X \pi}^{+} d p_{X \pi}^{-} d p_{\pi}^{-}}\right|_{p_{\pi}^{-}=z p_{X \pi}^{-}}\right|_{p_{X \pi}^{-}=m_{X \pi}^{2} / p_{X \pi}^{+}},
$$

where we indicate the two changes of variable explicitly. The integration boundaries are derived from Eq. (11).

In Sec VI we will illustrate how to extract from this doubly differential decay rate information about the standard parton fragmentation function $D_{u}^{\pi}$.

\section{PROPERTIES OF $\mathcal{G}$}

\section{A. Relations with the inclusive jet function, $J(s, \mu)$}

If we sum over all possible hadrons $h$ in the $X_{u} \rightarrow X h$ fragmentation process, then the fragmenting jet function can be related to the inclusive jet function $J_{u}(s, \mu)$ which is completely calculable in QCD perturbation theory. Consider the equality

$$
\sum_{h \in \mathcal{H}_{u}} \int d p_{h}^{-} \int d p_{h}^{+} \frac{d^{4} \Gamma}{d p_{X h}^{+} d p_{X h}^{-} d p_{h}^{-} d p_{h}^{+}}=\frac{d^{2} \Gamma}{d p_{X_{u}}^{+} d p_{X_{u}}^{-}}
$$

where the sum with $h \in \mathcal{H}_{u}$ is over all final states with an identified $h$ hadron fragmenting from the $u$-quark jet. The differential decay rate on the right-hand side involves the hadronic light-cone variables in the process $\bar{B} \rightarrow X_{u} \ell \bar{\nu}$. The sum takes $p_{X h}^{+} \rightarrow p_{X_{u}}^{+}$and comparing our Eq. (68) with the leading-order factorization theorem for inclusive $\bar{B} \rightarrow X_{u} \ell \bar{\nu}$ (see e.g. [38])

$$
\frac{d^{2} \Gamma}{d p_{X_{u}}^{+} d p_{X_{u}}^{-}}=16 \pi^{3} \Gamma_{0} H\left(m_{B}, p_{X_{u}}^{-}, p_{X_{u}}^{+}\right) p_{X_{u}}^{-} \int_{0}^{p_{X_{u}}^{+}} d k^{\prime+} J_{u}\left(p_{X_{u}}^{-}\left(p_{X_{u}}^{+}-k^{\prime+}\right), \mu\right) S\left(k^{\prime+}, \mu\right),
$$

one obtains [50]:

$$
\sum_{h \in \mathcal{H}_{u}} \int d z z \mathcal{G}_{j}^{h}\left(k^{+} p_{X h}^{-}, z, \mu\right)=2(2 \pi)^{3} J_{j}\left(k^{+} p_{X_{u}}^{-}, \mu\right)
$$

for $j=u$, where $J_{u}$ is the leading-order quark jet function. The factor $z$ under the integral is explained in Ref. [50], and is necessary to provide the correct symmetry factor for states with identical particles. As the notation indicates, Eq. (72) holds for other partons $j=$ $\{g, d, \bar{u}, \ldots\}$ as well. This relation between the fragmenting jet function $\mathcal{G}_{j}^{h}$ and the jet function is not surprising since the set of states $\left\{|X h\rangle_{h \in \mathcal{H}_{u}}\right\}$ is complete. The factor $2(2 \pi)^{3}$ is related to how we normalized $\mathcal{G}_{q}^{h}$ and incorporated the phase space for $h$. 


\section{B. Relations with the standard fragmentation function $D_{q}^{h}(x, \mu)$}

In the SCET notation, Eq. (77) can be written in terms of the collinear q-quark field

$$
D_{q}^{h}\left(\frac{p_{h}^{-}}{\omega}, \mu\right)=\pi \omega \int d p_{h}^{+} \frac{1}{4 N_{c}} \operatorname{Tr} \sum_{X} \not h\left\langle 0\left|\left[\delta_{\omega, \overline{\mathcal{P}}} \delta_{0, \mathcal{P}_{\perp}} \chi_{n}(0)\right]\right| X h\right\rangle\left\langle X h\left|\bar{\chi}_{n}(0)\right| 0\right\rangle
$$

since $\left|p_{h}^{\perp}\right| d\left|p_{h}^{\perp}\right|=\left(p_{h}^{-} / 2\right) d p_{h}^{+}$at a fixed value of $p_{h}^{-}$. Here $\mu$ is the $\overline{\mathrm{MS}}$ renormalization scale. According to Eq. (52), the integral of $\mathcal{G}_{q}^{h}$ over its first argument can be written as

$$
\begin{aligned}
& \int \frac{d k^{+}}{2 \pi} e^{-i k^{+} x^{-} / 2} \mathcal{G}_{q}^{h}\left(k^{+} \omega, \frac{p_{h}^{-}}{\omega}, \mu\right)= \\
= & \frac{1}{2} \int d p_{h}^{+} \int d x^{+} \int d^{2} x_{\perp} \frac{1}{4 N_{c}} \operatorname{Tr} \sum_{X} \not h\left\langle 0\left|\left[\delta_{\omega, \overline{\mathcal{P}}} \delta_{0, \mathcal{P}_{\perp}} \chi_{n}(x)\right]\right| X h\right\rangle\left\langle X h\left|\bar{\chi}_{n}(0)\right| 0\right\rangle .
\end{aligned}
$$

If we perform an operator product expansion on the right-hand-side of this equation we match onto a low energy matrix element that gives the fragmentation function in Eq. (173). Thus, $\mathcal{G}_{j}^{h}$ is given by the convolution of a perturbatively calculable $\mathcal{J}_{i j}$ and the standard parton fragmentation function. The result includes mixing between parton types:

$$
\mathcal{G}_{i}^{h}(s, z, \mu)=\sum_{j} \int_{z}^{1} \frac{d x}{x} \mathcal{J}_{i j}\left(s, \frac{z}{x}, \mu\right) D_{j}^{h}(x, \mu)\left[1+\mathcal{O}\left(\frac{\Lambda_{\mathrm{QCD}}^{2}}{s}\right)\right],
$$

where $i, j=\{u, d, g, \bar{u}, \ldots\}$. In Ref. [51] the concept of a quark "beam function" is discussed. It turns out that a quark beam function is the analog of $\mathcal{G}_{q}^{h}$, but with parton distributions in place of fragmentation functions (and an incoming proton in place of an outgoing pion). The derivation of the factorization theorem in Eq. (75) can be carried out in a manner analogous to the matching of the gluon beam function onto a gluon parton distribution, as derived in Ref. [52]. For the factorization theorem for the fragmenting jet function in Eq. (75), the Wilson coefficient $\mathcal{J}_{i j}$ describes the formation of a final state jet with invariant mass $s$ within which the nonperturbative, long-distance fragmentation process takes place.

At tree level Eq. (75) is easily verified. Using a free $q$-quark of momentum $p$ in place of $h$ in the final state in Eq. (75) (and denoting the label parts of $p^{\mu}$ by $p_{\ell}^{-}, p_{\ell}^{\perp}$ and the residual parts by $p_{r}^{\mu}$, and defining $\left.z=p^{-} / \omega\right)$, the partonic $\mathcal{G}$ is

$$
\begin{aligned}
\mathcal{G}_{q}^{\text {tree }}\left(k^{+} \omega, z\right) & =\int \frac{d p_{r}^{+}}{4} \int d x^{-} d x^{+} d^{2} x_{\perp} e^{i k^{+} x^{-} / 2} \frac{1}{4 N_{c}} \operatorname{Tr}\left[\not h \delta_{\omega, p_{\ell}^{-}} \delta_{0, p_{\ell}^{\perp}}\left\langle 0\left|\xi_{n}(x)\right| q(p)\right\rangle\left\langle q(p)\left|\bar{\xi}_{n}(0)\right| 0\right\rangle\right] \\
& =\sum_{p_{\ell}^{\perp}} \int \frac{d^{2} p_{r}^{\perp}}{4 \pi p_{\ell}^{-}} \int d x^{-} d x^{+} d^{2} x_{\perp} e^{i\left[\left(k^{+}-p_{\ell \perp}^{2} / p_{\ell}^{-}\right) x^{-} / 2-p_{r}^{-} x^{+} / 2-p_{r}^{\perp} \cdot x_{\perp}\right]} \delta_{\omega, p_{\ell}^{-}} \delta_{0, p_{\ell}^{\perp}} p_{\ell}^{-} \\
& =\sum_{p_{\ell}^{\perp}} \int \frac{d^{2} p_{r}^{\perp}}{4 \pi} 4(2 \pi)^{4} \delta\left(k^{+}-p_{\ell \perp}^{2} / p_{\ell}^{-}\right) \delta\left(p_{r}^{-}\right) \delta^{2}\left(p_{r}^{\perp}\right) \delta_{\omega, p_{\ell}^{-}} \delta_{0, p_{\ell}^{\perp}} \\
& =2(2 \pi)^{3} \delta\left(k^{+}\right) \delta\left(\omega-p^{-}\right)=2(2 \pi)^{3} \delta\left(k^{+} \omega\right) \delta(1-z) .
\end{aligned}
$$


In the second to last step we recombined the residuals and labels into the continuous $p^{-}$, via $\delta_{\omega, p_{\ell}^{-}} \delta\left(p_{r}^{-}\right)=\delta\left(\omega-p^{-}\right)$. The quark fragmentation function is $D_{q}^{\text {tree }}(z)=\delta(1-z)$. Since the Wilson coefficients $\mathcal{J}_{i j}$ are independent of the choice of states, the tree-level coefficient function can be identified as

$$
\mathcal{J}_{q q}^{\text {tree }}\left(k^{+} \omega, z / x, \mu\right)=2(2 \pi)^{3} \delta\left(k^{+} \omega\right) \delta(1-z / x),
$$

which satisfies Eq. (75). The one-loop calculation of $\mathcal{J}_{i j}$ is presented in Ref. [50].

\section{VI. $\quad D_{u}^{\pi}(z)$ FROM A DOUBLY DIFFERENTIAL DECAY RATE}

As a further consequence of our factorization formulae, we explore a strategy to extract from measurements of suitable differential $B$-decay rates the standard pion fragmentation function $D_{u}^{\pi}(z)$ for values of $z$ that are not too small, such as $z \gtrsim 0.5$. Ultimately we anticipate that fragmenting jet functions will be useful for many other processes (including hadron-hadron collisions) for which a factorization theorem like Eq. (65) can be derived involving $\mathcal{G}_{i}^{h}$. The phenomenology of $B$-decays is particularly instructive to this purpose since it allows to concentrate on single jet production avoiding the kinematical complications of more involved scattering processes.

For comparison with phenomenology, we are interested in the doubly differential decay rate in Eq.(69). We aim at writing a factorization formula of the type:

$$
\frac{d^{2} \Gamma^{\mathrm{cut}}}{d m_{X \pi}^{2} d z}=\Gamma_{0} \sum_{j=u, \bar{u}, d, g . .} \int_{z}^{1} \frac{d x}{x} \hat{H}_{u j}\left(m_{b}, m_{X \pi}^{2}, \frac{z}{x}, \mu\right) D_{j}^{\pi}(x, \mu)
$$

where $\hat{H}_{u j}$ is calculable in perturbation theory and the cut refers to a suitable interval in $p_{\mathbf{X}_{\pi}}^{+}$ over which we integrate. We shall argue that in the "shape function OPE" regime [32, 33] $\left(p_{X \pi}^{-} \gg p_{X \pi}^{+} \gg \Lambda_{\mathrm{QCD}}\right)$ it is possible to write a factorization formula involving $D_{u}^{\pi}$, which does not specify the invariant mass of the final-state jet.

According to the discussion in Ref. [38], the shape function can be written as a convolution when integrated over a large enough interval $[0, \Delta]$ such that perturbation theory is applicable at the scale $\Delta$ :

$$
S(\omega)=\int_{0}^{\infty} d \omega^{\prime} C_{0}\left(\omega-\omega^{\prime}\right) F\left(\omega^{\prime}\right)
$$

where $C_{0}$ is the $b$-quark matrix element of the shape function operator calculated in perturbation theory and $F$ is a non-perturbative function that can be determined by comparison with data. $F$ falls off exponentially for large $\omega^{\prime}$ and all its moments exist without a cutoff. 
Combining Eqs. (68), (69) and (175), the integration over $p_{X \pi}^{+}$leads to

$$
\begin{aligned}
\frac{d^{2} \Gamma^{\text {cut }}}{d m_{X \pi}^{2} d z}= & \Gamma_{0} \sum_{j=u, \bar{u}, d, g \ldots} \int_{z}^{1} \frac{d x}{x} D_{j}^{\pi}(x, \mu) \int_{m_{X \pi}^{2} / m_{B}}^{m_{X \pi}} d p_{X \pi}^{+} \frac{m_{X \pi}^{2}}{\left(p_{X \pi}^{+}\right)^{2}} H\left(m_{B}, \frac{m_{X \pi}^{2}}{p_{X \pi}^{+}}, p_{X \pi}^{+}, \mu\right) \\
& \times \int_{0}^{p_{X \pi}^{+}} d k^{+} \mathcal{J}_{u j}\left(k^{+} \frac{m_{X \pi}^{2}}{p_{X \pi}^{+}}, \frac{z}{x}, \mu\right) \int_{0}^{\infty} d \omega^{\prime} C_{0}\left(p_{X \pi}^{+}-k^{+}-\omega^{\prime}, \mu\right) F\left(\omega^{\prime}\right)
\end{aligned}
$$

if $p_{X \pi}^{+\max } \gg \Lambda_{\mathrm{QCD}}$. Let us now perform a Taylor expansion of the perturbative kernel $C_{0}$ around $\omega^{\prime}=0$ :

$$
C_{0}\left(p_{X \pi}^{+}-k^{+}-\omega^{\prime}\right)=C_{0}\left(p_{X \pi}^{+}-k^{+}\right)-\omega^{\prime} C_{0}^{\prime}\left(p_{X \pi}^{+}-k^{+}\right)+\ldots
$$

Since [38]

$$
\int_{0}^{\infty} d \omega^{\prime} F\left(\omega^{\prime}\right)=1 \quad \text { and } \quad \int_{0}^{\infty} d \omega^{\prime} \omega^{\prime n} F\left(\omega^{\prime}\right)=\mathcal{O}\left(\Lambda_{\mathrm{QCD}}^{n}\right)
$$

the $k^{+}$-convolution integral in Eq.

$$
\int_{0}^{p_{X \pi}^{+}} d k^{+} \mathcal{J}_{u j}\left(k^{+} \frac{m_{X \pi}^{2}}{p_{X \pi}^{+}}, \frac{z}{x}, \mu\right) C_{0}\left(p_{X \pi}^{+}-k^{+}, \mu\right)+\ldots
$$

where the dots indicate terms suppressed by increasing powers of $\Lambda_{\mathrm{QCD}} / p_{X \pi}^{+}$in the phasespace region where the jet becomes less collimated and increases its invariant mass $\left(p_{X \pi}^{+} \gg\right.$ $\left.\Lambda_{\mathrm{QCD}}\right)$. Hence, for $p_{X \pi}^{+\min } \gg \Lambda_{\mathrm{QCD}}$ and $p_{X \pi}^{+\max } \ll p_{X \pi}^{-}$, at leading order in the $\Lambda_{\mathrm{QCD}} / p_{X \pi^{-}}^{+}$ expansion,

$$
\begin{aligned}
\frac{d^{2} \Gamma^{\mathrm{cut}}}{d m_{X \pi}^{2} d z}= & \Gamma_{0} \sum_{j=u, \bar{u}, d, g \ldots} \int_{z}^{1} \frac{d x}{x} D_{j}^{\pi}(x, \mu) \int_{m_{X \pi}^{2} / m_{B}}^{m_{X \pi}} d p_{X \pi}^{+} \frac{m_{X \pi}^{2}}{\left(p_{X \pi}^{+}\right)^{2}} H\left(m_{B}, \frac{m_{X \pi}^{2}}{p_{X \pi}^{+}}, p_{X \pi}^{+}, \mu\right) \\
& \times \int_{0}^{p_{X \pi}^{+}} d k^{+} \mathcal{J}_{u j}\left(k^{+} \frac{m_{X \pi}^{2}}{p_{X \pi}^{+}}, \frac{z}{x}, \mu\right) C_{0}\left(p_{X \pi}^{+}-k^{+}, \mu\right) .
\end{aligned}
$$

By identifying

$$
\begin{aligned}
\hat{H}_{u j}\left(m_{B}, m_{X \pi}^{2}, \frac{z}{x}, \mu\right) \Leftrightarrow & \int_{m_{X \pi}^{2} / m_{B}}^{m_{X \pi}} d p_{X \pi}^{+} \frac{m_{X \pi}^{2}}{\left(p_{X \pi}^{+}\right)^{2}} H\left(m_{B}, \frac{m_{X \pi}^{2}}{p_{X \pi}^{+}}, p_{X \pi}^{+}, \mu\right) \\
& \times \int_{0}^{p_{X \pi}^{+}} d k^{+} \mathcal{J}_{u j}\left(k^{+} \frac{m_{X \pi}^{2}}{p_{X \pi}^{+}}, \frac{z}{x}, \mu\right) C_{0}\left(p_{X \pi}^{+}-k^{+}, \mu\right),
\end{aligned}
$$

we see that Eq. (78) is satisfied at leading order in SCET. Note that to obtain the complete inclusive $\hat{H}_{u j}$ there are additional hard corrections from processes beyond those treated in the jet-like region, so Eq. (85) does not give the complete expression for $\hat{H}_{u j}$. Following the same steps, one can also test the consistency with the factorized expression

$$
\frac{d^{3} \Gamma^{\text {cut }}}{d m_{X \pi}^{2} d z d E_{\ell}}=3 \Gamma_{0} \int_{z}^{1} \frac{d x}{x} \hat{H}_{u j}\left(m_{b}, m_{X \pi}^{2}, E_{\ell}, \frac{z}{x}, \mu\right) D_{j}^{\pi}(x, \mu),
$$

where $\bar{H}\left(m_{B}, p_{X \pi}^{-}=m_{X \pi}^{2} / p_{X \pi}^{+}, p_{X \pi}^{+}, E_{\ell}, \mu\right)$ in Eq. (64) replaces $H$ in Eq. (85). 


\section{CONCLUSIONS}

Using Soft-Collinear Effective Theory, we have derived leading-order factorization formulae for differential decay rates in the process $\bar{B} \rightarrow X h \ell \bar{\nu}$ where $h$ is a light, energetic hadron fragmenting from a measured $u$-quark jet. We obtained results for differential decay rates with various kinematic variables, for example

$$
\frac{d^{3} \Gamma}{d p_{X h}^{+} d p_{X h}^{-} d p_{h}^{-}}=\Gamma_{0} H\left(m_{B}, p_{X h}^{-}, p_{X h}^{+}, \mu\right) \int_{0}^{p_{X h}^{+}} d k^{+} \mathcal{G}_{u}^{h}\left(k^{+} p_{X \pi}^{-}, z, \mu\right) S\left(p_{X \pi}^{+}-k^{+}, \mu\right)
$$

where $\Gamma_{0}$ is a constant prefactor, $H$ encodes contributions from hard scales, $z=p_{h}^{-} / p_{X h}^{-}$ and $S$ is the leading-order shape function. $\mathcal{G}_{i}^{h}$ is the novel leading-order fragmenting jet function: at variance with the standard parton fragmentation function $D_{i}^{h}(z)$, it incorporates information about the invariant mass of the jet from which the detected hadron fragments.

We have also shown that it is possible to extract $D_{i}^{h}(z)$ from a suitable $\bar{B} \rightarrow X h \ell \bar{\nu}$ differential decay rate, for values of $z$ that are not too small, like $z \gtrsim 0.5$.

Moreover, our analysis implies that to obtain a factorization theorem for a semi-inclusive process where the hadron $h$ fragments from a jet, it is sufficient to take the factorization theorem for the corresponding inclusive case and make the replacement

$$
J_{j}\left(k^{+} \omega\right) \longrightarrow \frac{1}{2(2 \pi)^{3}} \mathcal{G}_{j}^{h}\left(k^{+} \omega, z\right) d z
$$

where $J_{j}$ is the inclusive jet function for parton $j$ and the additional phase space variable is $z=p_{h}^{-} / p_{X h}^{-}$, the momentum fraction of the hadron relative to the total large momentum of the $X h$ system. This replacement rule is consistent with integration over the phase space for $h$. Applying Eq. (88) to the factorization theorem given schematically in Eq. (4) we derive the following factorization formula for the doubly differential decay rate in the process $\bar{B} \rightarrow X K \gamma$ :

$$
\begin{aligned}
& \frac{d^{2} \Gamma}{d E_{\gamma} d z}=\frac{\Gamma_{0 s} m_{b}}{(2 \pi)^{3}} H_{s}\left(p_{X K}^{+}, \mu\right) \int_{0}^{p_{X K}^{+}} d k^{+} \mathcal{G}_{s}^{K}\left(k^{+} m_{b}, z, \mu\right) S\left(p_{X K}^{+}-k^{+}, \mu\right) \\
& =\frac{\Gamma_{0 s} m_{b}}{(2 \pi)^{3}} H_{s}\left(p_{X K}^{+}, \mu\right) \sum_{j} \int_{0}^{p_{X K}^{+}} d k^{+} \int_{z}^{1} \frac{d x}{x} \mathcal{J}_{s j}\left(k^{+} m_{b}, \frac{z}{x}, \mu\right) D_{j}^{K}(x, \mu) S\left(p_{X K}^{+}-k^{+}, \mu\right)
\end{aligned}
$$

with $p_{X K}^{+}=m_{B}-2 E_{\gamma}$, and the $\Gamma_{0 s}$ and $H_{s}$ are defined in Eq. (5) and Eq. (A1) of Ref. [38]. The soft function $S$ is the same one as in endpoint $\bar{B} \rightarrow X_{s} \gamma$. The jet Wilson coefficients $\mathcal{J}_{s j}$ are process independent and calculable in perturbation theory. At tree level:

$$
\mathcal{J}_{s s}^{\text {tree }}\left(k^{+} \omega, z / x, \mu\right)=2(2 \pi)^{3} \delta\left(k^{+} \omega\right) \delta(1-z / x) .
$$

Analogously, for the process $e^{+} e^{-} \rightarrow$ (dijets) $+h$ we apply Eq. (88) to the factorization 
theorem for $e^{+} e^{-} \rightarrow$ (dijets) in Eq. (3) to obtain the factorized differential cross-section

$$
\begin{aligned}
\frac{d^{3} \sigma}{d M^{2} d \bar{M}^{2} d z}= & \frac{\sigma_{0}}{2(2 \pi)^{3}} H_{2 \mathrm{jet}}(Q, \mu) \int_{-\infty}^{+\infty} d l^{+} d l^{-}\left[\mathcal{G}_{q}^{h}\left(M^{2}-Q l^{+}, z, \mu\right) J_{\bar{n}}\left(\bar{M}^{2}-Q l^{-}, \mu\right)+\right. \\
& \left.J_{n}\left(M^{2}-Q l^{+}, \mu\right) \mathcal{G}_{\bar{q}}^{h}\left(\bar{M}^{2}-Q l^{-}, z, \mu\right)\right] S_{2 \mathrm{jet}}\left(l^{+}, l^{-}, \mu\right) \\
= & \frac{\sigma_{0}}{2(2 \pi)^{3}} H_{2 \mathrm{jet}}(Q, \mu) \\
& \times \sum_{j} \int_{-\infty}^{+\infty} d l^{+} d l^{-} \int_{z}^{1} \frac{d x}{x}\left[\mathcal{J}_{q j}\left(M^{2}-Q l^{+}, \frac{z}{x}, \mu\right) J_{\bar{n}}\left(\bar{M}^{2}-Q l^{-}, \mu\right)+\right. \\
& \left.J_{n}\left(M^{2}-Q l^{+}, \mu\right) \mathcal{J}_{\bar{q} j}\left(\bar{M}^{2}-Q l^{-}, \frac{z}{x}, \mu\right)\right] D_{j}^{h}(x, \mu) S_{2 \mathrm{jet}}\left(l^{+}, l^{-}, \mu\right),
\end{aligned}
$$

where $\sigma_{0}$ is the tree level total cross-section which acts as a normalization factor, $Q$ is the center-of-mass energy, $M^{2}$ and $\bar{M}^{2}$ are hemisphere invariant masses for the two hemispheres perpendicular to the dijet thrust axis. Since here we assume that it is not known whether the hadron $h$ fragmented from the quark or antiquark initiated jet, we have a sum over both possibilities in the factorization theorem. For the definitions of $\sigma_{0}, H_{2 j e t}$, and $S_{2 j e t}$ see Ref. [4] whose notation we have followed.

The factorization formulae derived with our analysis should allow improved constraints on parton fragmentation functions to light hadrons, by allowing improved control over the fragmentation environment with the invariant mass measurement, as well as opening up avenues for fragmentation functions to be measured in new processes, such as $B$-decays. We also expect that further study based on the definition of the fragmenting jet function, will contribute to a better understanding of the relative roles of perturbative partonic shortdistance effects and non-perturbative hadronization in shaping jet properties and features.

\section{ACKNOWLEDGMENTS}

This work was supported in part by the Office of Nuclear Physics of the U.S. Department of Energy under the Contract DE-FG02-94ER40818, and by the Alexander von Humboldt foundation through a Feodor Lynen Fellowship (M.P.) and a Friedrich Wilhelm Bessel award (I.S.). We acknowledge discussions with F. D'Eramo, A. Jain, and W. Waalewijn.

[1] J. C. Collins, D. E. Soper, and G. Sterman, Adv. Ser. Direct. High Energy Phys. 5, 1 (1988), hep-ph/0409313.

[2] G. P. Korchemsky and G. Sterman, Nucl. Phys. B 555, 335 (1999), hep-ph/9902341.

[3] G. P. Korchemsky and S. Tafat, JHEP 10, 010 (2000), hep-ph/0007005].

[4] S. Fleming, A. H. Hoang, S. Mantry, and I. W. Stewart, Phys. Rev. D 77, 074010 (2008), hep-ph/0703207.

[5] M. D. Schwartz, Phys. Rev. D 77, 014026 (2008), arXiv:0709.2709]. 
[6] G. P. Korchemsky and G. Sterman, Phys. Lett. B340, 96 (1994).

[7] C. W. Bauer, D. Pirjol, and I. W. Stewart, Phys. Rev. D 65, 054022 (2002), hep-ph/0109045].

[8] C. W. Bauer, S. Fleming, and M. E. Luke, Phys. Rev. D 63, 014006 (2001), hep-ph/0005275].

[9] C. W. Bauer, S. Fleming, D. Pirjol, and I. W. Stewart, Phys. Rev. D 63, 114020 (2001), hep-ph/0011336.

[10] C. W. Bauer and I. W. Stewart, Phys. Lett. B 516, 134 (2001), hep-ph/0107001.

[11] J. C. Collins and D. E. Soper, Nucl. Phys. B194, 445 (1982).

[12] J. C. Collins, Nucl. Phys. B396, 161 (1993), hep-ph/9208213].

[13] A. H. Mueller, Phys. Rev. D18, 3705 (1978).

[14] H. Georgi and H. D. Politzer, Nucl. Phys. B136, 445 (1978).

[15] R. K. Ellis, H. Georgi, M. Machacek, H. D. Politzer, and G. G. Ross, Nucl. Phys. B152, 285 (1979).

[16] G. Curci, W. Furmanski, and R. Petronzio, Nucl. Phys. B175, 27 (1980).

[17] S. Kretzer, Phys. Rev. D62, 054001 (2000), hep-ph/0003177.

[18] B. A. Kniehl, G. Kramer, and B. Potter, Nucl. Phys. B582, 514 (2000), hep-ph/0010289].

[19] S. Albino, B. A. Kniehl, and G. Kramer, Nucl. Phys. B725, 181 (2005), hep-ph/0502188.

[20] M. Hirai, S. Kumano, T. H. Nagai, and K. Sudoh, Phys. Rev. D75, 094009 (2007), hep-ph/0702250.

[21] D. de Florian, R. Sassot, and M. Stratmann, Phys. Rev. D75, 114010 (2007), hep-ph/0703242.

[22] D. de Florian, R. Sassot, and M. Stratmann, Phys. Rev. D76, 074033 (2007), arXiv:0707.1506.

[23] S. Albino, B. A. Kniehl, and G. Kramer, Nucl. Phys. B803, 42 (2008), 0803.2768].

[24] A. H. Mueller, Phys. Rev. D9, 963 (1974).

[25] C. G. Callan, Jr. and M. L. Goldberger, Phys. Rev. D11, 1553 (1975).

[26] J. C. Collins and D. E. Soper, Nucl. Phys. B193, 381 (1981).

[27] BABAR Collaboration, B. Aubert et al., hep-ex/0408068.

[28] BABAR Collaboration, K. Tackmann, Eur. Phys. J. A38, 137 (2008), 0801.2985].

[29] Belle Collaboration, P. Urquijo et al., 0907.0379.

[30] A. K. Leibovich, I. Low, and I. Z. Rothstein, Phys. Lett. B486, 86 (2000), hep-ph/0005124.

[31] C. W. Bauer, M. Luke, and T. Mannel, Phys. Lett. B543, 261 (2002), hep-ph/0205150].

[32] C. W. Bauer and A. V. Manohar, Phys. Rev. D70, 034024 (2004), hep-ph/0312109.

[33] S. W. Bosch, B. O. Lange, M. Neubert, and G. Paz, Nucl. Phys. B699, 335 (2004), hep-ph/0402094.

[34] K. S. M. Lee and I. W. Stewart, Nucl. Phys. B721, 325 (2005), hep-ph/0409045.

[35] M. Beneke, F. Campanario, T. Mannel, and B. D. Pecjak, JHEP 06, 071 (2005), hep-ph/0411395.

[36] B. O. Lange, M. Neubert, and G. Paz, Phys. Rev. D72, 073006 (2005), hep-ph/0504071].

[37] F. J. Tackmann, Phys. Rev. D72, 034036 (2005), hep-ph/0503095.

[38] Z. Ligeti, I. W. Stewart, and F. J. Tackmann, Phys. Rev. D 78, 114014 (2008), arXiv:0807.1926.

[39] M. Procura, I. W. Stewart, and F. J. Tackmann, In preparation.

[40] C. W. Bauer, D. Pirjol, and I. W. Stewart, Phys. Rev. D 67, 071502 (2003), hep-ph/0211069.

[41] D. Pirjol and I. W. Stewart, Phys. Rev. D67, 094005 (2003).

[42] J. Chay and C. Kim, Phys. Rev. D65, 114016 (2002), [hep-ph/0201197].

[43] R. Bonciani and A. Ferroglia, JHEP 11, 065 (2008), 0809.4687. 
[44] H. M. Asatrian, C. Greub, and B. D. Pecjak, Phys. Rev. D78, 114028 (2008), 0810.0987].

[45] M. Beneke, T. Huber, and X. Q. Li, Nucl. Phys. B811, 77 (2009), 0810.1230].

[46] G. Bell, Nucl. Phys. B812, 264 (2009), 0810.5695].

[47] A. V. Manohar, T. Mehen, D. Pirjol, and I. W. Stewart, Phys. Lett. B539, 59 (2002).

[48] M. Neubert, Phys. Rev. D49, 4623 (1994), hep-ph/9312311.

[49] I. I. Y. Bigi, M. A. Shifman, N. G. Uraltsev, and A. I. Vainshtein, Int. J. Mod. Phys. A9, 2467 (1994), hep-ph/9312359].

[50] A. Jain, M. Procura, and W. J. Waalewijn, arXiv:1101.4953.

[51] I. W. Stewart, F. J. Tackmann, and W. J. Waalewijn, arXiv:0910.0467.

[52] S. Fleming, A. K. Leibovich, and T. Mehen, Phys. Rev. D74, 114004 (2006), hep-ph/0607121. 